\title{
Quantum inverse scattering method and generalizations of symplectic Schur functions and Whittaker functions
}

\author{
Kohei Motegi ${ }^{1 *}$ Kazumitsu Sakai ${ }^{2 \dagger}$ and Satoshi Watanabe ${ }^{3 \ddagger}$ \\ ${ }^{1}$ Faculty of Marine Technology, \\ Tokyo University of Marine Science and Technology, \\ Etchujima 2-1-6, Koto-Ku, Tokyo, 135-8533, Japan \\ ${ }^{2}$ Department of Physics, Tokyo University of Science, \\ Kagurazaka 1-3, Shinjuku-ku, Tokyo, 162-8601, Japan \\ ${ }^{3}$ Institute of physics, University of Tokyo, \\ Komaba 3-8-1, Meguro-ku, Tokyo 153-8902, Japan
}

December 23, 2019

\begin{abstract}
We introduce generalizations of type $C$ and $B$ ice models which were recently introduced by Ivanov and Brubaker-Bump-Chinta-Gunnells, and study in detail the partition functions of the models by using the quantum inverse scattering method. We compute the explicit forms of the wavefunctions and their duals by using the Izergin-Korepin technique, which can be applied to both models. For type $C$ ice, we show the wavefunctions are expressed using generalizations of the symplectic Schur functions. This gives a generalization of the correspondence by Ivanov. For type $B$ ice, we prove that the exact expressions of the wavefunctions are given by generalizations of the Whittaker functions introduced by Bump-Friedberg-Hoffstein. The special case is the correspondence conjectured by Brubaker-Bump-Chinta-Gunnells. We also show the factorized forms for the domain wall boundary partition functions for both models. As a consequence of the studies of the partition functions, we obtain dual Cauchy formulas for the generalized symplectic Schur functions and the generalized Whittaker functions.
\end{abstract}

\section{Introduction}

Integrable lattice models, which are a special class of mathematical models in statistical physics, have been playing important roles in the developments of combinatorics and representation theory in modern mathematics. For example, investigating the mathematical

\footnotetext{
${ }^{*}$ E-mail: kmoteg0@kaiyodai.ac.jp

${ }^{\dagger}$ E-mail: k.sakai@rs.tus.ac.jp

${ }^{\ddagger}$ E-mail: watanabe@gokutan.c.u-tokyo.ac.jp
} 
structure of the $R$-matrices and $L$-operators lead to the discovery of quantum groups [1, 2] and the developments of the quantum inverse scattering method [3, 4, 5].

From the point of view of statistical physics, the most important quantities are partition functions. Partition functions of integrable lattice models are global quantities consisting of $L$ operators and under specified boundary conditions. A particular class called the domain wall boundary partition functions [6, 7] have found applications to the enumeration of alternating sign matrices (see [8, 9] for examples) in the 1990s.

Partition functions are now gaining attention again in combinatorial representation theory. It has been realized that analyzing the details of partition functions lead us to the discovery and refinements of algebraic identities such as the Cauchy and Littlewood identities for various types of symmetric functions like the Schur, Grothendieck, Hall-Littlewood polynomials, noncommutative versions and so on. Recently, there are various studies on this topic, including [10, 11, 12, 13, 14, 15, 16, 17, 18, 19, 20, 21, 22, 23, 24, 25, 26, 27, 28, 29.

The most fundamental and important thing to accomplish the detailed study is to find the exact correspondence between the wavefunctions and the symmetric functions, which depends on the type of the local $L$-operators and the global boundary conditions. In the correspondence, the spectral parameters of the integrable lattice models play the role of symmetric variables of the corresponding symmetric functions.

One of the seminal works are by Brubaker-Bump-Friedberg [11], which they showed that the wavefunctions of some free-fermionic six-vertex model are given as the product of some factors and the Schur functions. A free parameter lives in the free-fermionic six-vertex model, which plays the role of refining the combinatorial formula for the Schur polynomials. The Tokuyama formula [30, 31, 32] is a combinatorial formula which gives a deformation of the Weyl character formula, and Brubaker-Bump-Friedberg found that the wavefunctions of the free-fermionic six-vertex model gives an integrable model realization of the Tokuyama formula. The gauge transformed version of the $L$-operator are related with the Felderhof model [33, 34, 35] or the Perk-Schultz model [36] which the underlying quantum group structure are the colored representation of $U_{q}\left(s l_{2}\right)$ [34, 35] or superalgebra representation [37].

Today, there are developments on studying the variations and generalizations of the correspondence. See [31, 38, 39, 40, 41, 42, 43, 44, 45, 46, 47, 48, 52, 53, 54, 55] for examples on this topic as well as former related works on this model. For example, the correspondence between the wavefunctions of the free-fermionic six-vertex models and the Schur functions was generalized to the factorial Schur functions by Bump-McNamara-Nakasuji [12] (including [13, 14] as special cases) by introducing inhomogeneous parameters in the quantum spaces. They also showed that comparing two expressions for the domain wall boundary partition functions, one can derive a dual Cauchy identity for the factorial Schur functions.

One of the most recent developments are to twist the higher rank Perk-Schultz model [36] to construct the metaplectic ice [48], as done by Brubaker-Buciumas-Bump, which gives combinatorial descriptions of metaplectic Whittaker functions. The dual version of the metaplectic ice giving the same partition functions as the original one was recently constructed by Brubaker-Buciumas-Bump-Gray [49]. There are also related works on Hecke modules [50, and the notion of metaplectic ice was extended to type $C$ by Gray [51] by introducing the metaplectic ice under reflecting boundary. There is also a recent work on understanding the partition functions of metaplectic objects in terms of vertex operators by Brubaker-BuciumasBump-Gustafsson [52]. Another development is the extension of the work by BrubakerBump-Friedberg, Bump-McNamara-Nakasuji to the elliptic case [29, 55] by introducing and 
computing the wavefunctions of the elliptic generalization of the Felderhof model and the Perk-Schultz model, introduced by Foda-Wheeler-Zuparic [56] (see also Deguchi-Akutsu [35]) and Okado [57, Deguchi-Fujii [58] and Deguchi-Martin [59].

As for variations of the correspondence, the seminal works are by Ivanov [43, 44] and Brubaker-Bump-Chinta-Gunnells [46] (see also Hamel-King [38, 39] for former related works), in which they studied the wavefunctions under reflecting boundary conditions. Ivanov [43, 44] introduced and studied the wavefunctions of the six-vertex model under reflecting boundary (type $C$ ice), which a diagonal boundary $K$-matrix is used at the boundary, and showed that the explicit forms of the wavefunctions are expressed as a product of factors and symplectic Schur functions. By using a different boundary $K$-matrix (type $B$ ice), Brubaker-BumpChinta-Gunnells [46] conjectured the explicit forms of the wavefunctions. They conjectured that the wavefunctions are given by the product of factors and the Whittaker functions introduced by Bump-Friedberg-Hoffstein [60].

In this paper, we introduce and study in detail generalizations of the partition functions of the free-fermionic six-vertex model under reflecting boundary, and derive algebraic identities for symmetric functions as a consequence of the detailed study. We use an $L$-operator [20, 54] which is a specialization of the one in Brubaker-Bump-Friedberg [11] and which is a oneparameter deformation of the one used in Bump-McNamara-Nakasuji [12] as the bulk weights, and deal with the partition functions under reflecting boundary, using two types of $K$-matrices as the boundary weights which were used in Brubaker-Bump-Chinta-Gunnells [46] and Ivanov [43, 44]. See also [31, 62, 63, 64, 65, 66, 67, 68, 69, 70, 71, 72, 73] for examples on works on the domain wall boundary partition functions under reflecting boundary of the $U_{q}\left(s l_{2}\right)$ six-vertex model and the free-fermionic six-vertex model, and extensions to the elliptic model and to the wavefunctions. The elliptic models were first analyzed for the basic domain wall boundary partition functions without reflecting boundary conditions in [74, 75, 76, .

We analyze the wavefunctions, the dual wavefunctions and the domain wall boundary partition functions in detail using the quantum inverse scattering method. We use the IzerginKorepin technique, which was originally initiated by Korepin [6] as a trick to characterize the properties of the polynomials representing the domain wall boundary partition functions of the $U_{q}\left(s l_{2}\right)$ six-vertex model. Izergin later found the explicit determinant representation (Izergin-Korepin determinant) in [7]. The Izergin-Korepin technique was applied to analyze variations of the domain wall boundary partition functions (see Tsuchiya and Kuperberg [9, 62 for examples on seminal works of variations), and the method was recently extended to the scalar products by Wheeler [61] and to the wavefunctions by one of the authors $[29,54,55]$.

We use the Izergin-Korepin technique developed recently for the wavefunctions to prove the exact correspondence between the wavefunctions and the symmetric functions. As for the generalization of the type $C$ ice by Ivanov [43, 44], the wavefunctions can be also analyzed using the argument of Ivanov which extends the one by Brubaker-Bump-Friedberg [11]. However, the argument does not lead to the final answer for type $B$ ice, as mentioned in the paper by Brubaker-Bump-Chinta-Gunnells [46]. We use the Izergin-Korepin technique for the analysis as this method is applicable to the type $B$ ice as well as the type $C$ ice. For the Izergin-Korepin technique to work, one needs to generalize the $L$-operator, which is the one used in this paper. We show the exact correspondences between the wavefunctions, the dual wavefunctions and the symmetric functions by using this technique, and the symmetric functions which appear are generalizations of the (factorial) symplectic Schur functions and 
the Bump-Friedberg-Hoffstein Whittaker functions [60] for the type $C$ ice and type $B$ ice, respectively. We also show the factorized expressions for the domain wall boundary partition functions by using the Izergin-Korepin technique. Combining the above correspondences, we derive dual Cauchy formulas for the generalized symplectic Schur functions and the generalized Bump-Friedberg-Hoffstein Whittaker functions, using the idea of Bump-McNamaraNakasuji [12].

This paper is organized as follows. In the next section, we introduce a generalized $L$ operator of the free-fermionic six-vertex model. In section 3, we introduce two types of the wavefunctions under reflecting boundary, based on two different types of diagonal $K$-matrices and call them as the type I wavefunctions and the type II wavefunctions.

Sections 4 and 5 are devoted to the detailed study of the type I wavefunctions. In section 4, we make the Izergin-Korepin analysis on the wavefunctions and their duals, and derive their explicit forms and show the correspondence with the generalized symplectic Schur functions. In section 5 , we prove the complete factorized form for the domain wall boundary partition functions by the Izergin-Korepin analysis, and derive the dual Cauchy formula for the generalized symplectic Schur functions as a consequence of evaluating the domain wall boundary partition functions in two ways.

In sections 6 and 7, we perform a similar analysis on the type II partition functions. We derive the exact correspondence between the wavefunctions, the dual wavefunctions of type II and the generalized Bump-Friedberg-Hoffstein Whittaker functions. We also derive the factorized form of the type II domain wall boundary partition functions, and derive the dual Cauchy formulas for the generalized Bump-Friedberg-Hoffstein Whittaker functions. Section 8 is devoted to conclusion. Some calculations are deferred to Appendix.

\section{The free-fermionic six-vertex model}

In this section, we introduce the $R$-matrix and the $L$-operator of the free-fermionic sixvertex model [20, 54, and explain that the $L$-operator $L_{a j}\left(z, t, \alpha_{j}, \gamma_{j}\right)$ (2.6) which we use as the bulk elements of the wavefunctions in this paper is a specialization of the one in Brubaker-Bump-Friedberg [11] and is a one-parameter deformation of the one used in BumpMcNamara-Nakasuji [12].

The $R$-matrix of the free-fermionic six-vertex model is given as

$$
R(z, p, q)=\left(\begin{array}{cccc}
1-p q z & 0 & 0 & 0 \\
0 & -p^{2}\left(1-p^{-1} q z\right) & 1-q^{2} & 0 \\
0 & \left(1-p^{2}\right) z & z-p^{-1} q & 0 \\
0 & 0 & 0 & z-p q
\end{array}\right)
$$

which acts on the tensor product $W \otimes W$ of the complex two-dimensional vector space $W=\mathbb{C}^{2}$ spanned by the "empty state" $|0\rangle=\left(\begin{array}{l}1 \\ 0\end{array}\right)$ and the "particle occupied state" $|1\rangle=\left(\begin{array}{l}0 \\ 1\end{array}\right)$. The generalized $R$-matrix (2.1) can be shown to satisfy the Yang-Baxter relation

$$
\begin{aligned}
& R_{12}\left(z_{1} / z_{2}, p_{1}, p_{1}\right) R_{13}\left(z_{1}, p_{1}, p_{2}\right) R_{23}\left(z_{2}, p_{1}, p_{2}\right) \\
& =R_{23}\left(z_{2}, p_{1}, p_{2}\right) R_{13}\left(z_{1}, p_{1}, p_{2}\right) R_{12}\left(z_{1} / z_{2}, p_{1}, p_{1}\right),
\end{aligned}
$$

holding in $\operatorname{End}\left(W_{1} \otimes W_{2} \otimes W_{3}\right)$. 
There is one explanation why one can introduce at least two parameters $p$ and $q$ besides the spectral parameter $z$ in the generalized $R$-matrix (2.1) from the point of view of quantum groups. There is a class of an exotic quantum group called the colored representation or the nilpotent representation [34, 35]. The colored representation is a finite-dimensional highest weight representation which exists when the parameter of the quantum group is fixed at roots of unity. Each colored representation space is allowed to have a free parameter, and since the $R$-matrix is understood as an intertwiner acting on the tensor product of two representation spaces, one can include two free parameters.

The two types of the Boltzmann weights in [11] can be given as reductions from the generalized $R$-matrix (2.1). The first type of the Boltzmann weight in [11] comes by specializing $q$ to $q=p$ :

$$
R(z, t)=R(z, p, p)=\left(\begin{array}{cccc}
1+t z & 0 & 0 & 0 \\
0 & t(1-z) & t+1 & 0 \\
0 & (t+1) z & z-1 & 0 \\
0 & 0 & 0 & z+t
\end{array}\right)
$$

where we set $t=-p^{2}$.

The second type of Boltzmann weight is given by taking $q=0$ :

$$
L(z, t)=R(z, p, 0)=\left(\begin{array}{cccc}
1 & 0 & 0 & 0 \\
0 & t & 1 & 0 \\
0 & (t+1) z & z & 0 \\
0 & 0 & 0 & z
\end{array}\right)
$$

Under this specialization, the generalized Yang-Baxter relation 2.2 is rewritten as

$$
R_{a b}\left(z_{1} / z_{2}, t\right) L_{a j}\left(z_{1}, t\right) L_{b j}\left(z_{2}, t\right)=L_{b j}\left(z_{2}, t\right) L_{a j}\left(z_{1}, t\right) R_{a b}\left(z_{1} / z_{2}, t\right),
$$

acting on $W_{a} \otimes W_{b} \otimes V_{j}$. Here, $V$ and $W$ are both complex two-dimensional vector spaces $V=W=\mathbb{C}^{2}$. By convention we call $W$ and $V$ the auxiliary space and quantum space, respectively. The operator 2.3 acting on the tensor product of two auxiliary spaces $W \otimes W$ is called the $R$-matrix, and the operator (2.4) acting on the tensor product of one auxiliary and one quantum spaces $W \otimes V$ is called the $L$-operator. A class of the Yang-Baxter relation (2.5) is usually referred to as the $R L L$ relation.

A certain class of partition functions which we call wavefunctions, which we describe in more detail in the next section, is constructed from the $L$-operator. It was shown by Brubaker-Bump-Friedberg [11] that the wavefunctions for the basic case (without reflecting boundary) constructed from the $L$-operator (2.4) are expressed in terms of Schur functions. Later, the construction was extended to the factorial Schur functions by Bump-McNamaraNakasuji [12. From the quantum integrable point of view, the crucial point to construct the factorial Schur functions was to generalize the $L$-operator $(2.4)$ by keeping the $R L L$ relation (2.5).

We find a further generalization of the $L$-operator [20, 54] satisfying the $R L L$ relation which is given by

$$
L_{a j}\left(z, t, \alpha_{j}, \gamma_{j}\right)=\left(\begin{array}{cccc}
1-\gamma_{j} z & 0 & 0 & 0 \\
0 & t+\gamma_{j} z & 1 & 0 \\
0 & (t+1) z & \alpha_{j}+\left(1-\alpha_{j} \gamma_{j}\right) z & 0 \\
0 & 0 & 0 & -t \alpha_{j}+\left(1-\alpha_{j} \gamma_{j}\right) z
\end{array}\right)
$$


acting on $W_{a} \otimes V_{j}$. The parameters $\alpha_{j}$ and $\gamma_{j}$ can be regarded as parameters associated with the quantum space $V_{j}$. See Figure 1 for a graphical representation of (2.6). Hereafter we call this $L$-operator type $\Gamma L$-operator. The $L$-operator whose basic wavefunctions give the factorial Schur functions [12] is a special limit of the generalized $L$-operator 2.6 .

$$
L_{a j}\left(z, t, \alpha_{j}\right)=L_{a j}\left(z, t, \alpha_{j}, 0\right)=\left(\begin{array}{cccc}
1 & 0 & 0 & 0 \\
0 & t & 1 & 0 \\
0 & (t+1) z & \alpha_{j}+z & 0 \\
0 & 0 & 0 & -t \alpha_{j}+z
\end{array}\right)
$$

Let us make some comments on the $L$-operator (2.6). The $L$-operator $(2.6)$ is in fact a special case of a class of $L$-operators satisfying the free-fermion condition and satisfying the $R S T$ relation of Theorems 3 and 4 in Brubaker-Bump-Friedberg [11] (see also Proposition 1 in Bump-McNamara-Nakasuji [12]). What we call as the $L$-operator or the vertex which the $L$-operator is associated with corresponds to the operators $S, T$ in Theorem 3 in [11], and the vertices with free-fermionic Boltzmann weights $v$ and $w$ in Proposition 1 in [12. The $R$-matrix we call in this paper or the vertex which the $R$-matrix is associated with essentially corresponds to the operator $R$ in Theorem 3 in [11], and the vertex with the Boltzmann weights $u$ in Proposition 1 in [12] (note that there is a freedom to multiply the $R$-matrix by overall factors which keeps the $R L L$ relation). For example, it is explained in the Proof of Lemma 1 in [12] that to check the $L$-operator (2.7) and the $R$-matrix (2.3) (multiplied by an overall factor) satisfy the relation

$$
a_{1}(u)=a_{1}(v) a_{2}(w)+b_{2}(v) b_{1}(w)
$$

which is one of the relations first stated in [11] $\left(a_{1}(u), a_{1}(v), a_{2}(w), b_{2}(v), b_{1}(w)\right.$ are notations used in [12]) is nothing but to check the relation

$$
t z_{i}+z_{k}=1 \cdot\left(z_{k}-t \alpha_{j}\right)+\left(z_{i}+\alpha_{j}\right) t
$$

For the case of the $L$-operator (2.6) which is a one-parameter deformation of the one (2.7), checking the relation now becomes to check the following relation holds:

$$
t z_{i}+z_{k}=\left(1-\gamma_{j} z_{i}\right)\left(-t \alpha_{j}+\left(1-\alpha_{j} \gamma_{j}\right) z_{k}\right)+\left(\alpha_{j}+\left(1-\alpha_{j} \gamma_{j}\right) z_{i}\right)\left(t+\gamma_{j} z_{k}\right) .
$$

One can easily see that this identity still holds. One can also check the other five relations in [11], [12. It is also easy to check that the $L$-operator (2.6) satisfy the free-fermion condition. We use the $L$-operator (2.6) since we investigate the partition functions in this paper by the Izergin-Korepin method which we view as functions in the variables $\gamma_{j}$ s.

\section{Type I and type II wavefunctions under reflecting boundary}

In this section, we introduce two types of the wavefunctions under reflecting boundary by using $K$-matrices of Ivanov [43, 44] and Brubaker-Bump-Chinta-Gunnells [46]. The wavefunctions are constructed from the double monodromy matrices. To introduce double monodromy matrices, we introduce the following another $L$-operator (we call it type $\Delta L$-operator 


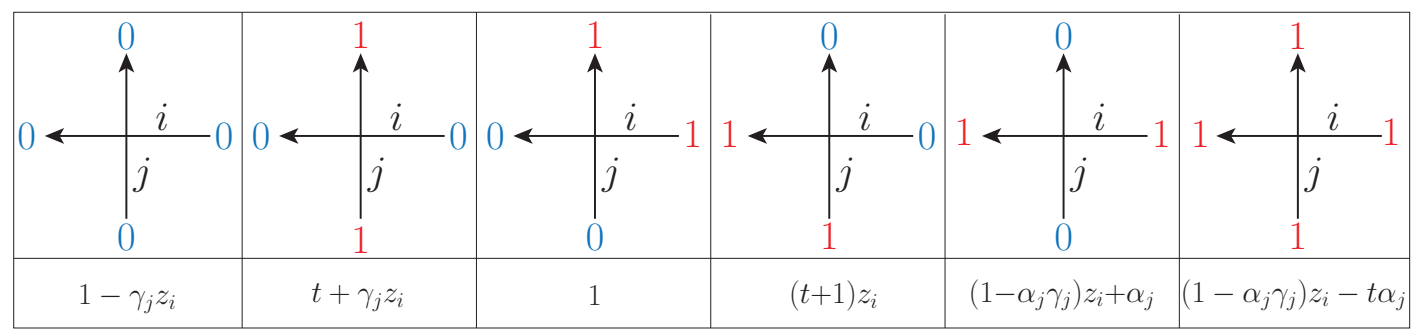

Figure 1: Type $\Gamma L$-operator (2.6).

$\widetilde{L}_{a j}\left(z, t, \alpha_{j}, \gamma_{j}\right)$ at the $j$ th site in the quantum space (Figure 2)

$$
\widetilde{L}_{a j}\left(z, t, \alpha_{j}, \gamma_{j}\right)=\left(\begin{array}{cccc}
\alpha_{j}+\left(1-\alpha_{j} \gamma_{j}\right) z & 0 & 0 & 0 \\
0 & t\left(1-\alpha_{j} \gamma_{j}\right) z-\alpha_{j} & 1 & 0 \\
0 & (t+1) z & 1-\gamma_{j} z & 0 \\
0 & 0 & 0 & t \gamma_{j} z+1
\end{array}\right) .
$$

Note that the type $\Gamma$ and $\Delta$ operators satisfy the following Yang-Baxter relations
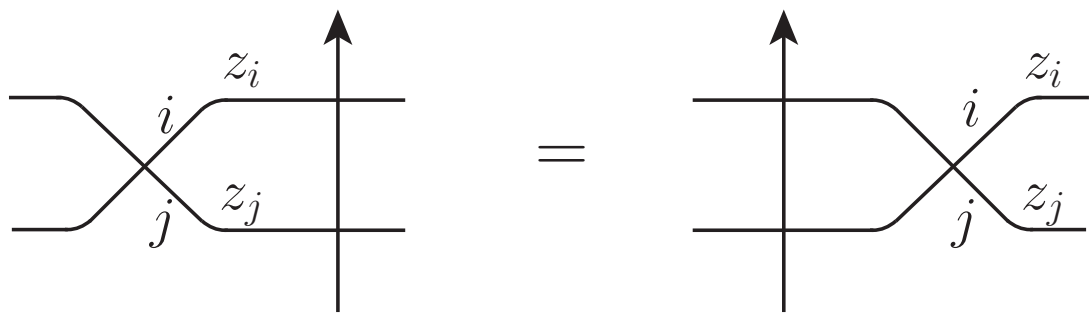

where the $R$-matrices are defined in Figure 3. At the boundary, we use boundary weights called as the $K$-matrix. We use two types of $K$-matrices in this paper. The first $K$-matrix $K_{a}^{\mathrm{I}}\left(z, t, \alpha_{0}, \gamma_{0}\right)$ is given by (Figure 4 )

$$
K_{a}^{\mathrm{I}}\left(z, t, \alpha_{0}, \gamma_{0}\right)=\left(\begin{array}{cc}
\left.1-\alpha_{0} \gamma_{0}\right) t z-\alpha_{0} & 0 \\
0 & \left(1-\alpha_{0} \gamma_{0}\right) z^{-1}+\alpha_{0}
\end{array}\right),
$$

where $\alpha_{0}, \gamma_{0}$ is a free parameter. (3.3) is a generalization of the one given by Ivanov. Setting $\alpha_{0}=\gamma_{0}=0,(3.3)$ reduces to the $K$-matrix used in [43, 44]. The $K$-matrix (3.3) satisfies the reflection equation [77]




We use another diagonal $K$-matrix in this paper. The second $K$-matrix $K_{a}^{\mathrm{II}}(z, t)$ is the one introduced by Brubaker-Bump-Chinta-Gunnells [46] (Figure 5)

$$
K_{a}^{\mathrm{II}}(z, t)=\left(\begin{array}{cc}
-\sqrt{-t} z^{1 / 2} & 0 \\
0 & z^{-1 / 2}
\end{array}\right)
$$

which also satisfies the reflection equation (3.4).

From the generalized $L$-operators (2.6) and (3.1), one constructs two types of monodromy matrices

$$
T_{a}(z,\{\alpha\},\{\gamma\})=L_{a M}\left(z^{-1}, t, \alpha_{M}, \gamma_{M}\right) \cdots L_{a 1}\left(z^{-1}, t, \alpha_{1}, \gamma_{1}\right)
$$

and

$$
\widetilde{T}_{a}(z,\{\alpha\},\{\gamma\})=\widetilde{L}_{a 1}\left(z, t, \alpha_{1}, \gamma_{1}\right) \cdots \widetilde{L}_{a M}\left(z, t, \alpha_{M}, \gamma_{M}\right),
$$

which act on $W_{a} \otimes\left(V_{1} \otimes \cdots \otimes V_{M}\right)$, We denote the matrix elements of the two monodromy matrices as

$$
\begin{aligned}
& A(z,\{\alpha\},\{\gamma\})={ }_{a}\left\langle 0\left|T_{a}(z,\{\alpha\},\{\gamma\})\right| 0\right\rangle_{a} \\
& B(z,\{\alpha\},\{\gamma\})={ }_{a}\left\langle 0\left|T_{a}(z,\{\alpha\},\{\gamma\})\right| 1\right\rangle_{a}
\end{aligned}
$$

and

$$
\begin{aligned}
& \widetilde{A}(z,\{\alpha\},\{\gamma\})={ }_{a}\left\langle 1\left|\widetilde{T}_{a}(z,\{\alpha\},\{\gamma\})\right| 1\right\rangle_{a}, \\
& \widetilde{B}(z,\{\alpha\},\{\gamma\})={ }_{a}\left\langle 0\left|\widetilde{T}_{a}(z,\{\alpha\},\{\gamma\})\right| 1\right\rangle_{a},
\end{aligned}
$$

where, $\{\alpha\}=\left\{\alpha_{1}, \ldots, \alpha_{M}\right\}$ and $\{\gamma\}=\left\{\gamma_{1}, \ldots, \gamma_{M}\right\}$.

Next, we introduce the double row $B$-operators. We define two types depending on which $K$-matrix we use at the boundary. For type I $K$-matrix $(3.3)$, we define the type I double row $B$-operator $\mathcal{B}^{\mathrm{I}}(z,\{\bar{\alpha}\},\{\bar{\gamma}\})$ as

$$
\begin{aligned}
\mathcal{B}^{\mathrm{I}}(z,\{\bar{\alpha}\},\{\bar{\gamma}\})= & \widetilde{B}(z,\{\alpha\},\{\gamma\})_{a}\left\langle 0\left|K_{a}^{\mathrm{I}}\left(z, t, \alpha_{0}, \gamma_{0}\right)\right| 0\right\rangle_{a} A(z,\{\alpha\},\{\gamma\}) \\
& +\widetilde{A}(z,\{\alpha\},\{\gamma\})_{a}\left\langle 1\left|K_{a}^{\mathrm{I}}\left(z, t, \alpha_{0}, \gamma_{0}\right)\right| 1\right\rangle_{a} B(z,\{\alpha\},\{\gamma\}) \\
=\{ & \left.\left(1-\alpha_{0} \gamma_{0}\right) t z-\alpha_{0}\right\} \widetilde{B}(z,\{\alpha\},\{\gamma\}) A(z,\{\alpha\},\{\gamma\}) \\
& +\left\{\left(1-\alpha_{0} \gamma_{0}\right) z^{-1}+\alpha_{0}\right\} \widetilde{A}(z,\{\alpha\},\{\gamma\}) B(z,\{\alpha\},\{\gamma\}) .
\end{aligned}
$$

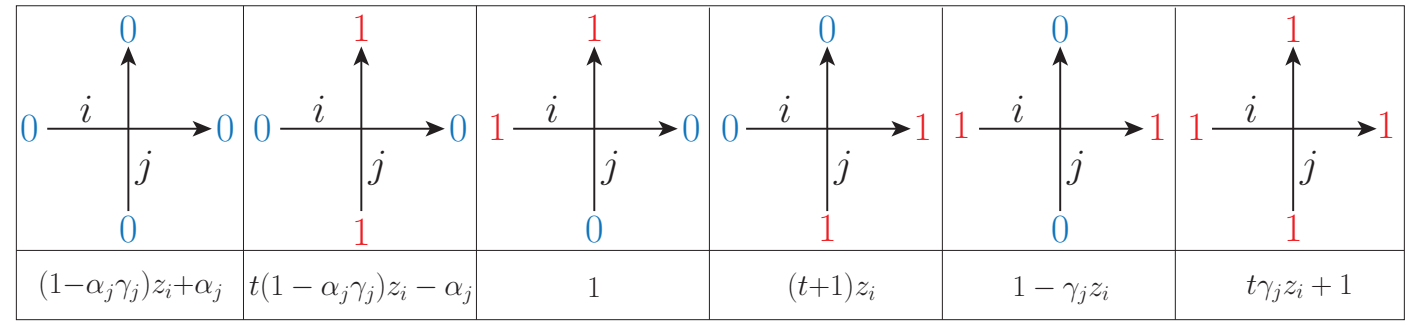

Figure 2: Type $\Delta L$-operator (3.1). 




$$
\left(z=z_{i} / z_{j}\right)
$$

Figure 3: $R$-matrices.

Here, the set of parameters $\{\bar{\alpha}\}$ and $\{\bar{\gamma}\}$ means $\{\bar{\alpha}\}=\left\{\alpha_{0}\right\} \sqcup\{\alpha\}$ and $\{\bar{\gamma}\}=\left\{\gamma_{0}\right\} \sqcup\{\gamma\}$, respectively.

For type II $K$-matrix (3.5), we similarly define $\mathcal{B}^{\mathrm{II}}(z,\{\alpha\},\{\gamma\})$ as

$$
\begin{aligned}
\mathcal{B}^{\mathrm{II}}(z,\{\alpha\},\{\gamma\})=\widetilde{B}(z,\{\alpha\},\{\gamma\})_{a}\left\langle 0\left|K_{a}^{\mathrm{II}}(z, t)\right| 0\right\rangle_{a} A(z,\{\alpha\},\{\gamma\}) & \\
& +\widetilde{A}(z,\{\alpha\},\{\gamma\})_{a}\left\langle 1\left|K_{a}^{\mathrm{II}}(z, t)\right| 1\right\rangle_{a} B(z,\{\alpha\},\{\gamma\}) \\
=-\sqrt{-t} z^{1 / 2} \widetilde{B}(z,\{\alpha\},\{\gamma\}) A(z,\{\alpha\},\{\gamma\}) & \\
& +z^{-1 / 2} \widetilde{A}(z,\{\alpha\},\{\gamma\}) B(z,\{\alpha\},\{\gamma\}) .
\end{aligned}
$$

See Figure 6 for graphical representations of the double row $B$-operators (3.10), (3.11).

We also introduce notations for special states in the tensor product of the Fock spaces



Figure 4: The type I $K$-matrix 3.3 . 
$V_{1} \otimes \cdots \otimes V_{M}$ and its dual $\left(V_{1} \otimes \cdots \otimes V_{M}\right)^{*}$ as

$$
\begin{array}{ll}
\left|0^{M}\right\rangle:=|0\rangle_{1} \otimes \cdots \otimes|0\rangle_{M}, \quad\left|1^{M}\right\rangle:=|1\rangle_{1} \otimes \cdots \otimes|1\rangle_{M}, \\
\left\langle 0^{M}\right|:={ }_{1}\langle 0| \otimes \cdots \otimes{ }_{M}\langle 0|, \quad & \left\langle 1^{M}\right|:={ }_{1}\langle 1| \otimes \cdots \otimes{ }_{M}\langle 1| .
\end{array}
$$

By acting the operators $\sigma_{j}^{+}$and $\sigma_{j}^{-}$defined by

$$
\begin{aligned}
& \sigma_{j}^{+}|1\rangle_{j}=|0\rangle_{j}, \quad \sigma_{j}^{+}|0\rangle_{j}=0, \quad{ }_{j}\langle 1| \sigma_{j}^{+}=0, \quad{ }_{j}\langle 0| \sigma_{j}^{+}={ }_{j}\langle 1|, \\
& \sigma_{j}^{-}|0\rangle_{j}=|1\rangle_{j}, \quad \sigma_{j}^{-}|1\rangle_{j}=0, \quad{ }_{j}\langle 0| \sigma_{j}^{-}=0, \quad{ }_{j}\langle 1| \sigma_{j}^{-}={ }_{j}\langle 0|,
\end{aligned}
$$

on $\left|0^{M}\right\rangle,\left\langle 0^{M}|,| 1^{M}\right\rangle$, we introduce states

$$
\left\langle x_{1} \cdots x_{N}\left|=\left\langle 0^{M}\left|\prod_{j=1}^{N} \sigma_{x_{j}}^{+}, \quad\right| x_{1} \cdots x_{N}\right\rangle=\prod_{j=1}^{N} \sigma_{x_{j}}^{-}\right| 0^{M}\right\rangle,
$$

for integers $x_{1}, \ldots, x_{N}$ satisfying $1 \leq x_{1}<x_{2}<\cdots<x_{N} \leq M$, and

$$
\left|\overline{x_{1}} \ldots \overline{x_{N}}\right\rangle=\prod_{j=1}^{N} \sigma_{\overline{x_{j}}}^{+}\left|1^{M}\right\rangle,
$$

for integers $\overline{x_{1}}, \ldots, \overline{x_{N}}$ satisfying $1 \leq \overline{x_{1}}<\overline{x_{2}}<\cdots<\overline{x_{N}} \leq M$.

We are now in a position to introduce the wavefunctions. We introduce the type I wavefunctions $\Phi_{M, N}^{\mathrm{I}}\left(z_{1}, \ldots, z_{N}\left|\gamma_{1}, \ldots, \gamma_{M}\right| x_{1}, \ldots, x_{N}\right)$ by acting the type I double-row $B$ operators $\mathcal{B}^{\mathrm{I}}\left(z_{j},\{\bar{\alpha}\},\{\bar{\gamma}\}\right)(j=1, \ldots, N)$ 3.10 on the state $\left|0^{M}\right\rangle$ and taking the inner product with $\left\langle x_{1} \cdots x_{N}\right|$ (Figure 7 ):

$$
\begin{aligned}
& \Phi_{M, N}^{\mathrm{I}}\left(z_{1}, \ldots, z_{N}\left|\gamma_{1}, \ldots, \gamma_{M}\right| x_{1}, \ldots, x_{N}\right) \\
& \quad=\left\langle x_{1} \cdots x_{N}\left|\mathcal{B}^{\mathrm{I}}\left(z_{1},\{\bar{\alpha}\},\{\bar{\gamma}\}\right) \cdots \mathcal{B}^{\mathrm{I}}\left(z_{N},\{\bar{\alpha}\},\{\bar{\gamma}\}\right)\right| 0^{M}\right\rangle .
\end{aligned}
$$

Likewise, we define the dual wavefunctions of type $\mathrm{I} \bar{\Phi}_{M, N}^{\mathrm{I}}\left(z_{1}, \ldots, z_{N}\left|\gamma_{1}, \ldots, \gamma_{M}\right| x_{1}, \ldots, x_{N}\right)$ as (Figure 8)

$$
\begin{aligned}
& \bar{\Phi}_{M, N}^{\mathrm{I}}\left(z_{1}, \ldots, z_{N}\left|\gamma_{1}, \ldots, \gamma_{M}\right| \overline{x_{1}}, \ldots, \overline{x_{N}}\right) \\
& \quad=\left\langle 1^{M}\left|\mathcal{B}^{\mathrm{I}}\left(z_{1},\{\bar{\alpha}\},\{\bar{\gamma}\}\right) \cdots \mathcal{B}^{\mathrm{I}}\left(z_{N},\{\bar{\alpha}\},\{\bar{\gamma}\}\right)\right| \overline{x_{1}} \cdots \overline{x_{N}}\right\rangle .
\end{aligned}
$$

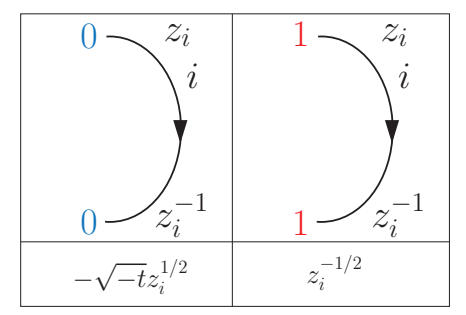

Figure 5: The type II $K$-matrix (3.5). 




Figure 6: The double row monodromy matrices $\mathcal{B}^{\mathrm{I}}(z,\{\bar{\alpha}\},\{\bar{\gamma}\})(3.10), \mathcal{B}^{\mathrm{II}}(z,\{\alpha\},\{\gamma\})$ 3.11).

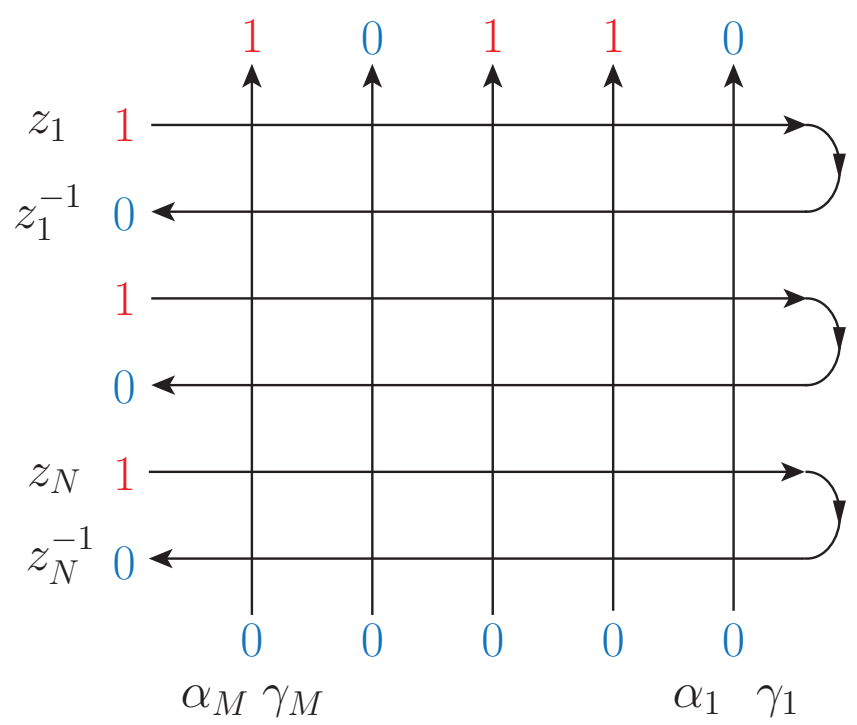

Figure 7: The wavefunctions $\left.\Phi_{M, N}^{\mathrm{I}}(3.17), \Phi_{M, N}^{\mathrm{II}} 3.19\right)$ under reflecting boundary. The figure illustrates the case $M=5, N=3, x_{1}=2, x_{2}=3, x_{3}=5$.

Similarly, we define the type II wavefunctions $\Phi_{M, N}^{\mathrm{II}}\left(z_{1}, \ldots, z_{N}\left|\gamma_{1}, \ldots, \gamma_{M}\right| x_{1}, \ldots, x_{N}\right)$ (Figure 7) and the dual wavefunctions $\bar{\Phi}_{M, N}^{\mathrm{II}}\left(z_{1}, \ldots, z_{N}\left|\gamma_{1}, \ldots, \gamma_{M}\right| \overline{x_{1}}, \ldots, \overline{x_{N}}\right)$ (Figure 8 by using the type II double-row $B$-operators $\mathcal{B}^{\mathrm{II}}\left(z_{N},\{\alpha\},\{\gamma\}\right)$ 3.11) as

$$
\begin{aligned}
& \Phi_{M, N}^{\mathrm{II}}\left(z_{1}, \ldots, z_{N}\left|\gamma_{1}, \ldots, \gamma_{M}\right| x_{1}, \ldots, x_{N}\right) \\
& \quad=\left\langle x_{1} \cdots x_{N}\left|\mathcal{B}^{\mathrm{II}}\left(z_{1},\{\alpha\},\{\gamma\}\right) \cdots \mathcal{B}^{\mathrm{II}}\left(z_{N},\{\alpha\},\{\gamma\}\right)\right| 0^{M}\right\rangle,
\end{aligned}
$$

and

$$
\begin{aligned}
& \bar{\Phi}_{M, N}^{\mathrm{II}}\left(z_{1}, \ldots, z_{N}\left|\gamma_{1}, \ldots, \gamma_{M}\right| \overline{x_{1}}, \ldots, \overline{x_{N}}\right) \\
& \quad=\left\langle 1^{M}\left|\mathcal{B}^{\mathrm{II}}\left(z_{1},\{\alpha\},\{\gamma\}\right) \cdots \mathcal{B}^{\mathrm{II}}\left(z_{N},\{\alpha\},\{\gamma\}\right)\right| \overline{x_{1}} \cdots \overline{x_{N}}\right\rangle .
\end{aligned}
$$

The wavefunctions of type I $\Phi_{M, N}^{\mathrm{I}}\left(z_{1}, \ldots, z_{N}\left|\gamma_{1}, \ldots, \gamma_{M}\right| x_{1}, \ldots, x_{N}\right)$ and type II $\Phi_{M, N}^{\mathrm{II}}\left(z_{1}, \ldots, z_{N}\left|\gamma_{1}, \ldots, \gamma_{M}\right| x_{1}, \ldots, x_{N}\right)$ are the partition functions which are generalizations of the ones introduced and studied by Ivanov and Brubaker-Bump-Chinta-Gunnells. The 


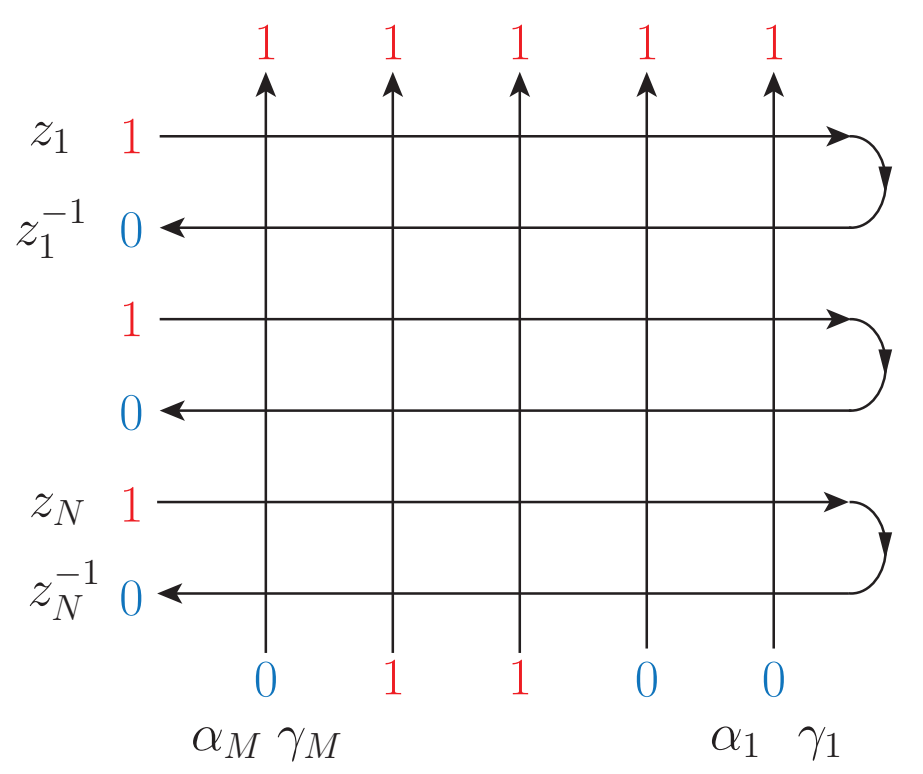

Figure 8: The dual wavefunctions $\bar{\Phi}_{M, N}^{\mathrm{I}}$ (3.18), $\bar{\Phi}_{M, N}^{\mathrm{II}}$ (3.20) under reflecting boundary. The figure illustrates the case $M=5, N=3, \overline{x_{1}}=1, \overline{x_{2}}=2, \overline{x_{3}}=5$.

special case $\alpha_{j}=0, \gamma_{j}=0(j=0, \ldots, M)$ of the type I wavefunctions was treated by Ivanov [43], in which the correspondence with the symplectic Schur functions were proven (the dual wavefunctions was treated recently in [67]). The special case $\alpha_{j}=0, \gamma_{j}=0(j=1, \ldots, M)$ of the type II wavefunctions were introduced by Brubaker-Bump-Chinta-Gunnells [46], and they conjectured the correspondence with the Bump-Friedberg-Hoffstein Whittaker functions [60.

In this paper, we compute the general case where there are no restrictions on the parameters $\alpha_{j}$ and $\gamma_{j}(j=0, \ldots, M)$. In the next two sections, we treat the wavefunctions, the dual wavefunctions and the domain wall boundary partition functions of type I. In sections 6 and 7 , we present the results for the type II wavefunctions.

\section{Type I wavefunctions and generalized symplectic Schur func- tions}

We derive the exact correspondence between the type I wavefunctions and the generalization of the symplectic Schur functions in this section. First, we compute the simplest case $N=1$. Next, we perform the Izergin-Korepin analysis, which is a trick to extract sufficiently many conditions to uniquely determine the wavefunctions (see [29, 54] for simpler examples without boundaries). Finally, we introduce the generalization of the symplectic Schur functions and show that the symmetric functions multiplied by some factors satisfy all the required properties extracted from the Izergin-Korepin analysis. 


\subsection{One particle case}

The simplest case $N=1$ of the type I wavefunctions can be calculated with the help of the following identity.

Lemma 4.1. The following identity holds:

$$
\begin{aligned}
\left(z-z^{-1}\right) & \sum_{j=1}^{x_{1}-1} \prod_{k=1}^{j-1}\left\{\alpha_{k}+\left(1-\alpha_{k} \gamma_{k}\right) z^{-1}\right\}\left(1-\gamma_{k} z\right) \prod_{k=j+1}^{x_{1}-1}\left\{\alpha_{k}+\left(1-\alpha_{k} \gamma_{k}\right) z\right\}\left(1-\gamma_{k} z^{-1}\right) \\
= & \prod_{k=1}^{x_{1}-1}\left\{\alpha_{k}+\left(1-\alpha_{k} \gamma_{k}\right) z\right\}\left(1-\gamma_{k} z^{-1}\right)-\prod_{k=1}^{x_{1}-1}\left\{\alpha_{k}+\left(1-\alpha_{k} \gamma_{k}\right) z^{-1}\right\}\left(1-\gamma_{k} z\right) .
\end{aligned}
$$

Proof. This can be proved by induction on $x_{1}$.

Proposition 4.2. The type I wavefunction $\Phi_{M, 1}^{\mathrm{I}}\left(z\left|\gamma_{1}, \ldots, \gamma_{M}\right| x_{1}\right)$ is explicitly expressed as

$$
\begin{aligned}
& \Phi_{M, 1}^{\mathrm{I}}\left(z\left|\gamma_{1}, \ldots, \gamma_{M}\right| x_{1}\right) \\
& \quad=\frac{1+t z^{2}}{z^{2}-1} \sum_{\tau= \pm 1} \tau \prod_{j=0}^{x_{1}-1}\left\{\alpha_{j}+\left(1-\alpha_{j} \gamma_{j}\right) z^{\tau}\right\} \prod_{j=x_{1}+1}^{M}\left(1-\gamma_{j} z^{\tau}\right) \prod_{j=1}^{M}\left(1-\gamma_{j} z^{-\tau}\right) .
\end{aligned}
$$

Proof. We can explicitly calculate $\Phi_{M, 1}^{\mathrm{I}}\left(z\left|\gamma_{1}, \ldots, \gamma_{M}\right| x_{1}\right)$ by decomposing as

$$
\begin{aligned}
\Phi_{M, 1}^{\mathrm{I}} & \left(z\left|\gamma_{1}, \ldots, \gamma_{M}\right| x_{1}\right) \\
= & \left\{\left(1-\alpha_{0} \gamma_{0}\right) t z-\alpha_{0}\right\}\left\langle x_{1}|\widetilde{B}(z,\{\alpha\},\{\gamma\})| 0^{M}\right\rangle\left\langle 0^{M}|A(z,\{\alpha\},\{\gamma\})| 0^{M}\right\rangle \\
& +\left\{\left(1-\alpha_{0} \gamma_{0}\right) z^{-1}+\alpha_{0}\right\}\left\langle x_{1}|\widetilde{A}(z,\{\alpha\},\{\gamma\})| x_{1}\right\rangle\left\langle x_{1}|B(z,\{\alpha\},\{\gamma\})| 0^{M}\right\rangle \\
& +\left\{\left(1-\alpha_{0} \gamma_{0}\right) z^{-1}+\alpha_{0}\right\} \sum_{j=1}^{x_{1}-1}\left\langle x_{1}|\widetilde{A}(z,\{\alpha\},\{\gamma\})| j\right\rangle\left\langle j|B(z,\{\alpha\},\{\gamma\})| 0^{M}\right\rangle,
\end{aligned}
$$

and computing the matrix elements of the monodromy matrices $\left\langle x_{1}|\widetilde{B}(z,\{\alpha\},\{\gamma\})| 0^{M}\right\rangle$, $\left\langle 0^{M}|A(z,\{\alpha\},\{\gamma\})| 0^{M}\right\rangle,\left\langle x_{1}|\widetilde{A}(z,\{\alpha\},\{\gamma\})| x_{1}\right\rangle,\left\langle x_{1}|B(z,\{\alpha\},\{\gamma\})| 0^{M}\right\rangle,\left\langle x_{1}|\widetilde{A}(z,\{\alpha\},\{\gamma\})| j\right\rangle$, $\left\langle j|B(z,\{\alpha\},\{\gamma\})| 0^{M}\right\rangle$ to get

$$
\begin{aligned}
& \Phi_{M, 1}^{\mathrm{I}}\left(z\left|\gamma_{1}, \ldots, \gamma_{M}\right| x_{1}\right)=\prod_{j=x_{1}+1}^{M}\left(1-\gamma_{j} z\right)\left(1-\gamma_{j} z^{-1}\right) \\
& \times\left[\left\{\left(1-\alpha_{0} \gamma_{0}\right) t z-\alpha_{0}\right\} \prod_{k=1}^{x_{1}-1}\left\{\left(1-\alpha_{k} \gamma_{k}\right) z+\alpha_{k}\right\} \prod_{k=1}^{x_{1}}\left(1-\gamma_{k} z^{-1}\right)\right. \\
& \quad+\left\{\left(1-\alpha_{0} \gamma_{0}\right) z^{-1}+\alpha_{0}\right\}\left(t \gamma_{x_{1}} z+1\right) \prod_{k=1}^{x_{1}-1}\left\{\left(1-\alpha_{k} \gamma_{k}\right) z^{-1}+\alpha_{k}\right\}\left(1-\gamma_{k} z\right) \\
& +(t+1) z\left\{\left(1-\alpha_{0} \gamma_{0}\right) z^{-1}+\alpha_{0}\right\} \sum_{j=1}^{x_{1}-1} \prod_{k=1}^{j-1}\left\{\left(1-\alpha_{k} \gamma_{k}\right) z^{-1}+\alpha_{k}\right\}\left(1-\gamma_{k} z\right) \\
& \left.\quad \times \prod_{k=j+1}^{x_{1}-1}\left\{\left(1-\alpha_{k} \gamma_{k}\right) z+\alpha_{k}\right\} \prod_{k=j+1}^{x_{1}}\left(1-\gamma_{k} z^{-1}\right)\right]
\end{aligned}
$$


Using the identity (4.1) in Lemma 4.1, one can show that the right hand side of (4.4) can be expressed as 4.2 .

We can similarly calculate the type I dual wavefunction $\bar{\Phi}_{M, 1}^{\mathrm{I}}\left(z\left|\gamma_{1}, \ldots, \gamma_{M}\right| \overline{x_{1}}\right)$. We state the result below.

Proposition 4.3. The type I dual wavefunction $\bar{\Phi}_{M, 1}^{\mathrm{I}}\left(z\left|\gamma_{1}, \ldots, \gamma_{M}\right| \overline{x_{1}}\right)$ is explicitly expressed as

$$
\begin{aligned}
& \bar{\Phi}_{M, 1}^{\mathrm{I}}\left(z\left|\gamma_{1}, \ldots, \gamma_{M}\right| \overline{x_{1}}\right)=\frac{t^{M}\left(1+t z^{2}\right)}{t^{2} z^{2}-1} \\
& \quad \times \sum_{\tau= \pm 1} \tau \prod_{j=0}^{\overline{x_{1}}-1}\left\{-\alpha_{j}+\left(1-\alpha_{j} \gamma_{j}\right)(t z)^{\tau}\right\} \prod_{j=\overline{x_{1}}+1}^{M}\left(1+\gamma_{j}(t z)^{\tau}\right) \prod_{j=1}^{M}\left\{1+\gamma_{j}(t z)^{-\tau}\right\} .
\end{aligned}
$$

\subsection{Izergin-Korepin analysis}

In this subsection, we use the Izergin-Korepin technique [6, 7, 29] and extract the properties of the type I wavefunctions which uniquely define them.

Proposition 4.4. The type I wavefunctions $\Phi_{M, N}^{\mathrm{I}}\left(z_{1}, \ldots, z_{N}\left|\gamma_{1}, \ldots, \gamma_{M}\right| x_{1}, \ldots, x_{N}\right)$ satisfies the following properties.

(1) When $x_{N}=M$, the type I wavefunctions $\Phi_{M, N}^{\mathrm{I}}\left(z_{1}, \ldots, z_{N}\left|\gamma_{1}, \ldots, \gamma_{M}\right| x_{1}, \ldots, x_{N}\right)$ is a polynomial of degree $2 N-1$ in $\gamma_{M}$.

(2) The following form

$$
\frac{\Phi_{M, N}^{\mathrm{I}}\left(z_{1}, \ldots, z_{N}\left|\gamma_{1}, \ldots, \gamma_{M}\right| x_{1}, \ldots, x_{N}\right)}{\prod_{j=1}^{N} z_{j}^{j-1-N}\left(1+t z_{j}^{2}\right) \prod_{1 \leq j<k \leq N}\left(1+t z_{j} z_{k}\right)\left(1+t z_{j} z_{k}^{-1}\right)},
$$

is symmetric with respect to $z_{1}, \ldots, z_{N}$, and is invariant under the exchange $z_{i} \longleftrightarrow z_{i}^{-1}$ for $i=1, \ldots, N$.

(3) The following recursive relations between the type I wavefunctions hold if $x_{N}=M$ (Figure 9):

$$
\begin{aligned}
\left.\Phi_{M, N}^{\mathrm{I}}\left(z_{1}, \ldots, z_{N}\left|\gamma_{1}, \ldots, \gamma_{M}\right| x_{1}, \ldots, x_{N}\right)\right|_{\gamma_{M}=z_{N}} & \\
= & \prod_{j=1}^{N}\left(t z_{N} z_{j}+1\right) \prod_{j=1}^{N-1}\left(t+z_{N} z_{j}^{-1}\right) \prod_{j=0}^{M-1}\left\{\left(1-\alpha_{j} \gamma_{j}\right) z_{N}^{-1}+\alpha_{j}\right\} \prod_{j=1}^{M-1}\left(1-\gamma_{j} z_{N}\right) \\
& \times \Phi_{M-1, N-1}^{\mathrm{I}}\left(z_{1}, \ldots, z_{N-1}\left|\gamma_{1}, \ldots, \gamma_{M-1}\right| x_{1}, \ldots, x_{N-1}\right) .
\end{aligned}
$$

If $x_{N} \neq M$, the following factorizations hold for the type I wavefunctions (Figure 10):

$$
\begin{aligned}
\Phi_{M, N}^{\mathrm{I}}\left(z_{1}, \ldots, z_{N}\left|\gamma_{1}, \ldots, \gamma_{M}\right| x_{1}, \ldots, x_{N}\right) \\
\quad=\prod_{j=1}^{N}\left(1-\gamma_{M} z_{j}\right)\left(1-\gamma_{M} z_{j}^{-1}\right) \Phi_{M-1, N}^{\mathrm{I}}\left(z_{1}, \ldots, z_{N}\left|\gamma_{1}, \ldots, \gamma_{M-1}\right| x_{1}, \ldots, x_{N}\right) .
\end{aligned}
$$


(4) The following holds for the case $N=1, x_{1}=M$ :

$$
\begin{aligned}
& \Phi_{M, 1}^{\mathrm{I}}\left(z\left|\gamma_{1}, \ldots, \gamma_{M}\right| M\right) \\
& \quad=\frac{1+t z^{2}}{z^{2}-1} \sum_{\tau= \pm 1} \tau \prod_{j=0}^{M-1}\left\{\alpha_{j}+\left(1-\alpha_{j} \gamma_{j}\right) z^{\tau}\right\} \prod_{j=1}^{M}\left(1-\gamma_{j} z^{-\tau}\right) .
\end{aligned}
$$



Figure 9: A graphical description of the relation (4.7). one can see that if one sets $\gamma_{M}$ to $\gamma_{M}=z_{N}$, all the $L$-operators at the leftmost column and the bottom double-row get frozen.

Proof. Property (1) can be shown in a standard way using a graphical representation of the wavefunctions.

Property (2) can be proved in the same way with Ivanov [43, 44], since the generalized $L$-operators (2.6), (3.1) and the generalized $K$-matrix (3.3) satisfy certain lemmas called the "caduceus relations", "fish relations", and the arguments built out of the lemmas work as well (see Appendix A for details). There is another argument to prove the invariance under the exchange $z_{i} \longleftrightarrow z_{i}^{-1}$ for $i=1, \ldots, N$ by using the argument applied to the dual wavefunctions in [67].

Property (4) is a special case $x_{1}=M$ of 4.2 in Proposition 4.2 which is already proven in the previous subsection.

The way to prove the two relations in Property (3) is also standard in the Izergin-Korepin analysis. We use the power of the graphical representation of the wavefunctions (Figures 9 and 10 . If $x_{N}=M$, one sees that after the specialization $\gamma_{M}=z_{N}$, the leftmost column and the bottom row gets frozen. The product of the matrix elements of the $L$-operators coming 
from the frozen part

$$
\prod_{j=1}^{N}\left(t z_{N} z_{j}+1\right) \prod_{j=1}^{N-1}\left(t+z_{N} z_{j}^{-1}\right) \prod_{j=0}^{M-1}\left\{\left(1-\alpha_{j} \gamma_{j}\right) z_{N}^{-1}+\alpha_{j}\right\} \prod_{j=1}^{M-1}\left(1-\gamma_{j} z_{N}\right),
$$

which, multiplied by the remaining part $\Phi_{M-1, N-1}^{\mathrm{I}}\left(z_{1}, \ldots, z_{N-1}\left|\gamma_{1}, \ldots, \gamma_{M-1}\right| x_{1}, \ldots, x_{N-1}\right)$, gives the specialization of $\Phi_{M, N}^{\mathrm{I}}\left(z_{1}, \ldots, z_{N}\left|\gamma_{1}, \ldots, \gamma_{M}\right| x_{1}, \ldots, x_{N}\right)$ at $\gamma_{M}=z_{N}$, i.e., 4.7) follows.

If $x_{N} \neq M$, one sees that the leftmost column is already frozen without imposing any specialization on $\gamma_{M}$, which gives the factor $\prod_{j=1}^{N}\left(1-\gamma_{M} z_{j}\right)\left(1-\gamma_{M} z_{j}^{-1}\right)$. The remaining part is $\Phi_{M-1, N}^{\mathrm{I}}\left(z_{1}, \ldots, z_{N}\left|\gamma_{1}, \ldots, \gamma_{M-1}\right| x_{1}, \ldots, x_{N}\right)$ and one concludes that the wavefunctions $\Phi_{M, N}^{\mathrm{I}}\left(z_{1}, \ldots, z_{N}\left|\gamma_{1}, \ldots, \gamma_{M}\right| x_{1}, \ldots, x_{N}\right)$ is the product of $\prod_{j=1}^{N}\left(1-\gamma_{M} z_{j}\right)\left(1-\gamma_{M} z_{j}^{-1}\right)$ and $\Phi_{M-1, N}^{\mathrm{I}}\left(z_{1}, \ldots, z_{N}\left|\gamma_{1}, \ldots, \gamma_{M-1}\right| x_{1}, \ldots, x_{N}\right)$, hence one gets 4.8.

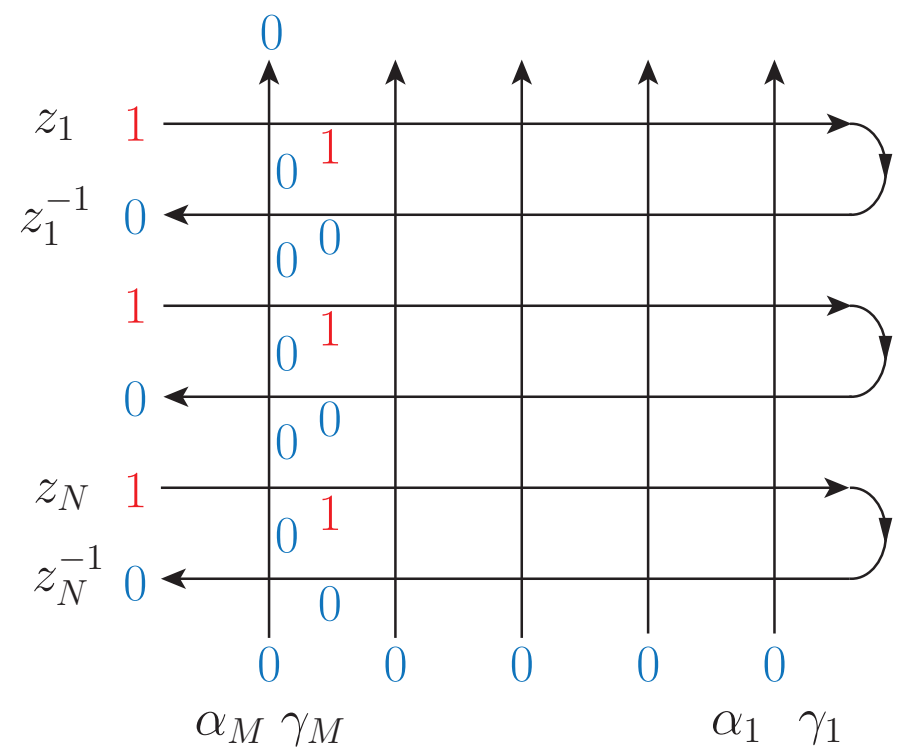

Figure 10: A graphical description of the factorization relation 4.8. One can see that all the $L$-operators at the leftmost column are frozen.

We also list the property for the type I dual wavefunctions.

Proposition 4.5. The type I dual wavefunctions $\bar{\Phi}_{M, N}^{\mathrm{I}}\left(z_{1}, \ldots, z_{N}\left|\gamma_{1}, \ldots, \gamma_{M}\right| \overline{x_{1}}, \ldots, \overline{x_{N}}\right)$ satisfies the following properties.

(1) When $\overline{x_{N}}=M$, the type I dual wavefunctions $\bar{\Phi}_{M, N}^{\mathrm{I}}\left(z_{1}, \ldots, z_{N}\left|\gamma_{1}, \ldots, \gamma_{M}\right| \overline{x_{1}}, \ldots, \overline{x_{N}}\right)$ is a polynomial of degree $2 N-1$ in $\gamma_{M}$.

(2) The following form

$$
\frac{\bar{\Phi}_{M, N}^{\mathrm{I}}\left(z_{1}, \ldots, z_{N}\left|\gamma_{1}, \ldots, \gamma_{M}\right| x_{1}, \ldots, x_{N}\right)}{\prod_{j=1}^{N} z_{j}^{j-1-N}\left(1+t z_{j}^{2}\right) \prod_{1 \leq j<k \leq N}\left(1+t z_{j} z_{k}\right)\left(1+t z_{j} z_{k}^{-1}\right)},
$$


is symmetric with respect to $z_{1}, \ldots, z_{N}$, and is invariant under the exchange $z_{i} \longleftrightarrow z_{i}^{-1}$ for $i=1, \ldots, N$.

(3) The following recursive relations between the type I dual wavefunctions hold if $\overline{x_{N}}=M$ :

$$
\begin{aligned}
\left.\bar{\Phi}_{M, N}^{\mathrm{I}}\left(z_{N}, \ldots, z_{1}\left|\gamma_{1}, \ldots, \gamma_{M}\right| \overline{x_{1}}, \ldots, \overline{x_{N}}\right)\right|_{\gamma_{M}=-t^{-1} z_{N}^{-1}} \\
=\prod_{j=1}^{N}\left(1+\frac{1}{t z_{N} z_{j}}\right) \prod_{j=1}^{N-1}\left(1+\frac{z_{j}}{t z_{N}}\right) \prod_{j=0}^{M-1}\left\{t\left(1-\alpha_{j} \gamma_{j}\right) z_{N}-\alpha_{j}\right\} \prod_{j=1}^{M-1}\left(t+\gamma_{j} z_{N}^{-1}\right) \\
\quad \times \bar{\Phi}_{M-1, N-1}^{\mathrm{I}}\left(z_{N-1}, \ldots, z_{1}\left|\gamma_{1}, \ldots, \gamma_{M-1}\right| \overline{x_{1}}, \ldots, \overline{x_{N-1}}\right) .
\end{aligned}
$$

If $\overline{x_{N}} \neq M$, the following factorizations hold for the type I dual wavefunctions:

$$
\begin{aligned}
& \bar{\Phi}_{M, N}^{\mathrm{I}}\left(z_{1}, \ldots, z_{N}\left|\gamma_{1}, \ldots, \gamma_{M}\right| \overline{x_{1}}, \ldots, \overline{x_{N}}\right) \\
& \quad=\prod_{j=1}^{N}\left(1+t \gamma_{M} z_{j}\right)\left(t+\gamma_{M} z_{j}^{-1}\right) \bar{\Phi}_{M-1, N}^{\mathrm{I}}\left(z_{1}, \ldots, z_{N}\left|\gamma_{1}, \ldots, \gamma_{M-1}\right| \overline{x_{1}}, \ldots, \overline{x_{N}}\right) .
\end{aligned}
$$

(4) The following holds for the case $N=1, \overline{x_{1}}=M$ :

$$
\begin{aligned}
& \bar{\Phi}_{M, 1}^{\mathrm{I}}\left(z\left|\gamma_{1}, \ldots, \gamma_{M}\right| M\right) \\
& \quad=\frac{t^{M}\left(1+t z^{2}\right)}{t^{2} z^{2}-1} \sum_{\tau= \pm 1} \tau \prod_{j=0}^{M-1}\left\{-\alpha_{j}+\left(1-\alpha_{j} \gamma_{j}\right)(t z)^{\tau}\right\} \prod_{j=1}^{M}\left\{1+\gamma_{j}(t z)^{-\tau}\right\} .
\end{aligned}
$$

We can prove Proposition 4.5 in a similar way with Proposition 4.4 . Note that the ordering of the spectral parameters in the dual wavefunctions in 4.12 are $z_{N}, \ldots, z_{1}$ (the ordering is $z_{1}, \ldots, z_{N}$ in the wavefunctions in (4.7)).

\subsection{Generalized symplectic Schur functions}

In this subsection, we first introduce the following symmetric functions which generalize the symplectic Schur functions.

Definition 4.6. We define the generalized symplectic Schur functions to be the following determinant:

$$
s p_{\lambda}\left(\{z\}_{N}|\{\bar{\alpha}\}|\{\bar{\gamma}\}\right)=\frac{G_{\lambda+\delta}\left(\{z\}_{N}|\{\bar{\alpha}\}|\{\bar{\gamma}\}\right)}{\operatorname{det}_{N}\left(z_{k}^{N-j+1}-z_{k}^{-N+j-1}\right)} .
$$

Here, $\{z\}_{N}=\left\{z_{1}, \ldots, z_{N}\right\}$ is a set of symmetric variables, $\{\bar{\alpha}\}$ and $\{\bar{\gamma}\}$ are sets of variables $\{\bar{\alpha}\}=\left\{\alpha_{0}, \alpha_{1}, \ldots, \alpha_{M}\right\}$ and $\{\bar{\gamma}\}=\left\{\gamma_{0}, \gamma_{1}, \ldots, \gamma_{M}\right\}, \lambda$ denotes a Young diagram $\lambda=\left(\lambda_{1}, \lambda_{2}, \ldots, \lambda_{N}\right)$ with weakly decreasing non-negative integers $\lambda_{1} \geq \lambda_{2} \geq \cdots \geq \lambda_{N} \geq 0$, and $\delta=(N-1, N-2, \ldots, 0) . G_{\mu}\left(\{z\}_{N}|\{\bar{\alpha}\}|\{\bar{\gamma}\}\right)$ is an $N \times N$ determinant

$$
G_{\mu}\left(\{z\}_{N}|\{\bar{\alpha}\}|\{\bar{\gamma}\}\right)=\operatorname{det}_{N}\left(g_{\mu_{j}}\left(z_{k}|\{\bar{\alpha}\}|\{\bar{\gamma}\}\right)-g_{\mu_{j}}\left(z_{k}^{-1}|\{\bar{\alpha}\}|\{\bar{\gamma}\}\right)\right),
$$

where

$$
g_{\mu}(z|\{\bar{\alpha}\}|\{\bar{\gamma}\})=\prod_{j=0}^{\mu}\left\{\alpha_{j}+\left(1-\alpha_{j} \gamma_{j}\right) z\right\} \prod_{j=\mu+2}^{M}\left(1-\gamma_{j} z\right) \prod_{j=1}^{M}\left(1-\gamma_{j} z^{-1}\right) .
$$


One can see from the definition 4.15 that the generalized symplectic Schur functions are symmetric functions with respect to the variables $z_{1}, \ldots, z_{N}$, and are invariant under the exchange $z_{i} \longleftrightarrow z_{i}^{-1}$ for $i=1, \ldots, N$. If one sets $\alpha_{j}=\gamma_{j}=0(j=0, \ldots, M)$, the generalized symplectic Schur functions reduce to the ordinary symplectic Schur functions.

Now we state the correspondence between the type I wavefunctions and the generalized symplectic Schur functions.

Theorem 4.7. The type I wavefunctions $\Phi_{M, N}^{\mathrm{I}}\left(z_{1}, \ldots, z_{N}\left|\gamma_{1}, \ldots, \gamma_{M}\right| x_{1}, \ldots, x_{N}\right)$ are explicitly expressed using the generalized symplectic Schur functions as

$$
\begin{aligned}
& \Phi_{M, N}^{\mathrm{I}}\left(z_{1}, \ldots, z_{N}\left|\gamma_{1}, \ldots, \gamma_{M}\right| x_{1}, \ldots, x_{N}\right) \\
& \quad=\prod_{j=1}^{N} z_{j}^{j-1-N}\left(1+t z_{j}^{2}\right) \prod_{1 \leq j<k \leq N}\left(1+t z_{j} z_{k}\right)\left(1+t z_{j} z_{k}^{-1}\right) s p_{\lambda}\left(\{z\}_{N}|\{\bar{\alpha}\}|\{\bar{\gamma}\}\right),
\end{aligned}
$$

under the relation $\lambda_{j}=x_{N-j+1}-N+j-1(j=1, \ldots, N)$.

The type I dual wavefunctions $\bar{\Phi}_{M, N}^{\mathrm{I}}\left(z_{1}, \ldots, z_{N}\left|\gamma_{1}, \ldots, \gamma_{M}\right| \overline{x_{1}}, \ldots, \overline{x_{N}}\right)$ are explicitly expressed using the generalized symplectic Schur functions as

$$
\begin{aligned}
& \quad \bar{\Phi}_{M, N}^{\mathrm{I}}\left(z_{1}, \ldots, z_{N}\left|\gamma_{1}, \ldots, \gamma_{M}\right| \overline{x_{1}}, \ldots, \overline{x_{N}}\right) \\
& \quad=t^{N(M-N)} \prod_{j=1}^{N} z_{j}^{j-1-N}\left(1+t z_{j}^{2}\right) \prod_{1 \leq j<k \leq N}\left(1+t z_{j} z_{k}\right)\left(1+t z_{j} z_{k}^{-1}\right) s p_{\bar{\lambda}}\left(\{t z\}_{N}|\{-\bar{\alpha}\}|\{-\bar{\gamma}\}\right),
\end{aligned}
$$

under the relation $\overline{\lambda_{j}}=\overline{x_{N-j+1}}-N+j-1(j=1, \ldots, N)$ and the symmetric variables are $\{t z\}_{N}=\left\{t z_{1}, \ldots, t z_{N}\right\}$. Moreover, the signs of the parameters of the generalized symplectic Schur functions in the right hand side of 4.19) are now inverted simultaneously: $\{-\bar{\alpha}\}=$ $\left\{-\alpha_{0},-\alpha_{1}, \ldots,-\alpha_{M}\right\}$ and $\{-\bar{\gamma}\}=\left\{-\gamma_{0},-\gamma_{1}, \ldots,-\gamma_{M}\right\}$.

The correspondences 4.18 ) and 4.19 are generalizations of the one by Ivanov in [43, 44] and one of the authors in [67]. One way to prove these correspondences is to adopt the argument by Brubaker-Bump-Friedberg [11] which they viewed the wavefunctions of the freefermionic six-vertex model without reflecting boundary as polynomials in $t$ and studied its properties to find the exact correspondence with the Schur functions. The argument works for this case as well. However, we use the Izergin-Korepin technique, since this argument works for the type II wavefunctions as well, in which case viewing as a function of $t$ can extract several properties but does not lead to the final form, as mentioned in Brubaker-BumpChinta-Gunnells [46]. Note that the Izergin-Korepin technique views the wavefunctions as functions of the parameter $\gamma_{M}$ associated with the quantum space $V_{M}$.

Proof. Let us show the correspondence 4.18). 4.19) can be proved in the same way (See Appendix for the detailed calculations). We have to show that the right hand side of 4.18 satisfies all the Properties in Proposition 4.4 .

It is first easy to see that Property (2) holds, since the right hand side of 4.18 divided by the factor $\prod_{j=1}^{N} z_{j}^{j-1-N}\left(1+t z_{j}^{2}\right) \prod_{1 \leq j<k \leq N}\left(1+t z_{j} z_{k}\right)\left(1+t z_{j} z_{k}^{-1}\right)$ is nothing but the generalized symplectic Schur functions $\operatorname{sp}_{\lambda}\left(\{\bar{z}\}_{N}|\{\bar{\alpha}\}|\{\bar{\gamma}\}\right)$ which is symmetric with respect to the variables $z_{1}, \ldots, z_{N}$ and is invariant under the exchange $z_{i} \longleftrightarrow z_{i}^{-1}$ for $i=1, \ldots, N$. 
Next, using the following factorization

$$
\operatorname{det}_{N}\left(z_{k}^{N-j+1}-z_{k}^{-N+j-1}\right)=(-1)^{N} \prod_{j=1}^{N} z_{j}^{j-1-N}\left(1-z_{j}^{2}\right) \prod_{1 \leq j<k \leq N}\left(1-z_{j} z_{k}\right)\left(1-z_{j} z_{k}^{-1}\right)
$$

and the definition of the determinant and the correspondence between the position of particles $\{x\}$ and the Young diagrams $\{\lambda\}$, we rewrite the right hand side of (4.18) as

$$
\begin{aligned}
F_{M, N}^{\mathrm{I}}\left(z_{1}, \ldots, z_{N}\left|\gamma_{1}, \ldots, \gamma_{M}\right| x_{1}, \ldots, x_{N}\right) \\
:=\frac{\prod_{j=1}^{N} z_{j}^{j-1-N}\left(1+t z_{j}^{2}\right) \prod_{1 \leq j<k \leq N}\left(1+t z_{j} z_{k}\right)\left(1+t z_{j} z_{k}^{-1}\right)}{(-1)^{N} \prod_{j=1}^{N} z_{j}^{j-1-N}\left(1-z_{j}^{2}\right) \prod_{1 \leq j<k \leq N}\left(1-z_{j} z_{k}\right)\left(-1+z_{j} z_{k}^{-1}\right)} \\
\quad \times \sum_{\sigma \in S_{N}} \sum_{\tau_{1}= \pm 1, \ldots, \tau_{N}= \pm 1}(-1)^{\sigma}(-1)^{|\tau|} \prod_{j=1}^{N} \prod_{k=0}^{x_{j}-1}\left\{\alpha_{k}+\left(1-\alpha_{k} \gamma_{k}\right) z_{\sigma(j)}^{\tau_{\sigma(j)}}\right\} \\
\quad \times \prod_{j=1}^{N} \prod_{k=x_{j}+1}^{M}\left(1-\gamma_{k} z_{\sigma(j)}^{\tau_{\sigma(j)}}\right) \prod_{j=1}^{N} \prod_{k=1}^{M}\left(1-\gamma_{k} z_{\sigma(j)}^{-\tau_{\sigma(j)}}\right),
\end{aligned}
$$

where $|\tau|$ denotes the number of $\tau_{j}$ 's satisfying $\tau_{j}=-1$. Note that the factor $(-1)^{N(N-1) / 2}$ appears when we write down the determinant in (4.17) in terms of the position of the particle $\{x\}$ instead of the Young diagrams $\{\lambda\}$.

It can be easily seen by rewriting the right hand side of (4.18) as 4.21) that it satisfies Property (4).

Property (1) is also easy to see from the expression (4.21). If $x_{N}=M$, one can see that the factors

$$
\prod_{j=1}^{N} \prod_{k=x_{j}+1}^{M}\left(1-\gamma_{k} z_{\sigma(j)}^{\tau_{\sigma(j)}}\right) \prod_{j=1}^{N} \prod_{k=1}^{M}\left(1-\gamma_{k} z_{\sigma(j)}^{-\tau_{\sigma(j)}}\right)
$$

which contain $\gamma_{M}$ in each summands become

$$
\prod_{j=1}^{N-1} \prod_{k=x_{j}+1}^{M}\left(1-\gamma_{k} z_{\sigma(j)}^{\tau_{\sigma(j)}}\right) \prod_{j=1}^{N} \prod_{k=1}^{M}\left(1-\gamma_{k} z_{\sigma(j)}^{-\tau_{\sigma(j)}}\right)
$$

from which one concludes that the degree with respect to $\gamma_{M}$ is $2 N-1$.

Let us prove that the functions $F_{M, N}^{\mathrm{I}}\left(z_{1}, \ldots, z_{N}\left|\gamma_{1}, \ldots, \gamma_{M}\right| x_{1}, \ldots, x_{N}\right)$ satisfy Property (3). We first treat the case $x_{N}=M$. Specializing $\gamma_{M}$ to $\gamma_{M}=z_{N}$, we find that only the summands satisfying $\sigma(N)=N, \tau_{N}=-1$ in 4.21 survive. Making use of this observation, 
we rewrite $\left.F_{M, N}^{\mathrm{I}}\left(z_{1}, \ldots, z_{N}\left|\gamma_{1}, \ldots, \gamma_{M}\right| x_{1}, \ldots, x_{N}\right)\right|_{\gamma_{M}=z_{N}}$ as

$$
\begin{aligned}
\left.F_{M, N}^{\mathrm{I}}\left(z_{1}, \ldots, z_{N}\left|\gamma_{1}, \ldots, \gamma_{M}\right| x_{1}, \ldots, x_{N}\right)\right|_{\gamma_{M}=z_{N}} \\
=-\frac{1+t z_{N}^{2}}{1-z_{N}^{2}} \frac{\prod_{j=1}^{N-1}\left(1+t z_{j} z_{N}\right)\left(1+t z_{j} z_{N}^{-1}\right)}{\prod_{j=1}^{N-1}\left(1-z_{j} z_{N}\right)\left(-1+z_{j} z_{N}^{-1}\right)} \\
\times \frac{\prod_{j=1}^{N-1}\left(1+t z_{j}^{2}\right) \prod_{1 \leq j<k \leq N-1}\left(1+t z_{j} z_{k}\right)\left(1+t z_{j} z_{k}^{-1}\right)}{(-1)^{N-1} \prod_{j=1}^{N-1}\left(1-z_{j}^{2}\right) \prod_{1 \leq j<k \leq N-1}\left(1-z_{j} z_{k}\right)\left(-1+z_{j} z_{k}^{-1}\right)} \\
\times \sum_{\sigma \in S_{N-1}} \sum_{1= \pm 1, \ldots, \tau_{N-1}= \pm 1}(-1)(-1)^{\sigma}(-1)^{|\tau|} \\
\times \prod_{j=1}^{N-1} \prod_{k=0}^{x_{j}-1}\left\{\alpha_{k}+\left(1-\alpha_{k} \gamma_{k}\right) z_{\sigma(j)}^{\tau_{\sigma(j)}}\right\} \prod_{k=0}^{M-1}\left\{\alpha_{k}+\left(1-\alpha_{k} \gamma_{k}\right) z_{N}^{-1}\right\} \\
\quad \times \prod_{j=1}^{N-1} \prod_{k=x_{j}+1}^{M-1}\left(1-\gamma_{k} z_{\sigma(j)}^{\tau_{\sigma(j)}}\right) \prod_{j=1}^{N-1}\left(1-z_{N} z_{\sigma(j)}^{\tau_{\sigma(j)}}\right) \\
\quad \times\left(1-z_{N}^{2}\right) \prod_{k=1}^{M-1}\left(1-\gamma_{k} z_{N}\right) \prod_{j=1}^{N-1}\left(1-z_{N} z_{\sigma(j)}^{-\tau_{\sigma(j)}}\right) \prod_{j=1}^{N-1} \prod_{k=1}^{M-1}\left(1-\gamma_{k} z_{\sigma(j)}^{-\tau_{\sigma(j)}}\right) .
\end{aligned}
$$

Using the obvious identity

$$
\prod_{j=1}^{N-1}\left(1-z_{N} z_{\sigma(j)}^{\tau_{\sigma(j)}}\right)\left(1-z_{N} z_{\sigma(j)}^{-\tau_{\sigma(j)}}\right)=\prod_{j=1}^{N-1}\left(1-z_{N} z_{j}\right)\left(1-z_{N} z_{j}^{-1}\right),
$$

and after some calculations, one sees that 4.24 can be simplified as

$$
\begin{aligned}
&\left.F_{M, N}^{\mathrm{I}}\left(z_{1}, \ldots, z_{N}\left|\gamma_{1}, \ldots, \gamma_{M}\right| x_{1}, \ldots, x_{N}\right)\right|_{\gamma_{M}=z_{N}} \\
&=\prod_{j=1}^{N}\left(t z_{N} z_{j}+1\right) \prod_{j=1}^{N-1}\left(t+z_{N} z_{j}^{-1}\right) \prod_{j=0}^{M-1}\left(\left(1-\alpha_{j} \gamma_{j}\right) z_{N}^{-1}+\alpha_{j}\right) \prod_{j=1}^{M-1}\left(1-\gamma_{j} z_{N}\right) \\
& \times \frac{\prod_{j=1}^{N-1} z_{j}^{j-1-(N-1)}\left(1+t z_{j}^{2}\right) \prod_{1 \leq j<k \leq N-1}\left(1+t z_{j} z_{k}\right)\left(1+t z_{j} z_{k}^{-1}\right)}{(-1)^{N-1} \prod_{j=1}^{N-1} z_{j}^{j-1-(N-1)}\left(1-z_{j}^{2}\right) \prod_{1 \leq j<k \leq N-1}\left(1-z_{j} z_{k}\right)\left(-1+z_{j} z_{k}^{-1}\right)} \\
& \times \sum_{\sigma \in S_{N-1} \tau_{1}= \pm 1, \ldots, \tau_{N-1}= \pm 1}(-1)^{\sigma}(-1)^{|\tau|} \prod_{j=1}^{N-1} \prod_{k=0}^{x_{j}-1}\left\{\alpha_{k}+\left(1-\alpha_{k} \gamma_{k}\right) z_{\sigma(j)}^{\tau_{\sigma(j)}}\right\} \\
& \times \prod_{j=1}^{N-1} \prod_{k=x_{j}+1}^{M-1}\left(1-\gamma_{k} z_{\sigma(j)}^{\tau_{\sigma(j)}}\right) \prod_{j=1}^{N-1} \prod_{k=1}^{M-1}\left(1-\gamma_{k} z_{\sigma(j)}^{-\tau_{\sigma(j)}}\right) \\
&= \prod_{j=1}^{N}\left(t z_{N} z_{j}+1\right) \prod_{j=1}^{N-1}\left(t+z_{N} z_{j}^{-1}\right) \prod_{j=0}^{M-1}\left\{\left(1-\alpha_{j} \gamma_{j}\right) z_{N}^{-1}+\alpha_{j}\right\} \prod_{j=1}^{M-1}\left(1-\gamma_{j} z_{N}\right) \\
& \times F_{M-1, N-1}^{\mathrm{I}}\left(z_{1}, \ldots, z_{N-1}\left|\gamma_{1}, \ldots, \gamma_{M-1}\right| x_{1}, \ldots, x_{N-1}\right),
\end{aligned}
$$

hence it is shown that $F_{M, N}^{\mathrm{I}}\left(z_{1}, \ldots, z_{N}\left|\gamma_{1}, \ldots, \gamma_{M}\right| x_{1}, \ldots, x_{N}\right)$ satisfies Property (3) for the case $x_{N}=M$. 
Property (3) for the case $x_{N} \neq M$ is much easier to prove. We just rewrite the functions $F_{M, N}^{\mathrm{I}}\left(z_{1}, \ldots, z_{N}\left|\gamma_{1}, \ldots, \gamma_{M}\right| x_{1}, \ldots, x_{N}\right)$ as

$$
\begin{aligned}
F_{M, N}^{\mathrm{I}}\left(z_{1}, \ldots, z_{N}\left|\gamma_{1}, \ldots, \gamma_{M}\right| x_{1}, \ldots, x_{N}\right) \\
=\frac{\prod_{j=1}^{N} z_{j}^{j-1-N}\left(1+t z_{j}^{2}\right) \prod_{1 \leq j<k \leq N}\left(1+t z_{j} z_{k}\right)\left(1+t z_{j} z_{k}^{-1}\right)}{(-1)^{N} \prod_{j=1}^{N} z_{j}^{j-1-N}\left(1-z_{j}^{2}\right) \prod_{1 \leq j<k \leq N}\left(1-z_{j} z_{k}\right)\left(-1+z_{j} z_{k}^{-1}\right)} \\
\quad \times \sum_{\sigma \in S_{N}} \sum_{\tau_{1}= \pm 1, \ldots, \tau_{N}= \pm 1}(-1)^{\sigma}(-1)^{|\tau|} \prod_{j=1}^{N} \prod_{k=0}^{x_{j}-1}\left\{\alpha_{k}+\left(1-\alpha_{k} \gamma_{k}\right) z_{\sigma(j)}^{\tau_{\sigma(j)}}\right\} \\
\quad \times \prod_{j=1}^{N} \prod_{k=x_{j}+1}^{M-1}\left\{1-\gamma_{k} z_{\sigma(j)}^{\tau_{\sigma(j)}}\right\} \prod_{j=1}^{N} \prod_{k=1}^{M-1}\left\{1-\gamma_{k} z_{\sigma(j)}^{-\tau_{\sigma(j)}}\right\} \\
\quad \times \prod_{j=1}^{N}\left(1-\gamma_{M} z_{\sigma(j)}^{\tau_{\sigma(j)}}\right) \prod_{j=1}^{N}\left(1-\gamma_{M} z_{\sigma(j)}^{-\tau_{\sigma(j)}}\right)
\end{aligned}
$$

and use the identity

$$
\prod_{j=1}^{N}\left(1-\gamma_{M} z_{\sigma(j)}^{\tau_{\sigma(j)}}\right) \prod_{j=1}^{N}\left(1-\gamma_{M} z_{\sigma(j)}^{-\tau_{\sigma(j)}}\right)=\prod_{j=1}^{N}\left(1-\gamma_{M} z_{j}\right) \prod_{j=1}^{N}\left(1-\gamma_{M} z_{j}^{-1}\right),
$$

to get

$$
\begin{aligned}
F_{M, N}^{\mathrm{I}}( & \left.z_{1}, \ldots, z_{N}\left|\gamma_{1}, \ldots, \gamma_{M}\right| x_{1}, \ldots, x_{N}\right) \\
= & \frac{\prod_{j=1}^{N} z_{j}^{j-1-N}\left(1+t z_{j}^{2}\right) \prod_{1 \leq j<k \leq N}\left(1+t z_{j} z_{k}\right)\left(1+t z_{j} z_{k}^{-1}\right)}{(-1)^{N} \prod_{j=1}^{N} z_{j}^{j-1-N}\left(1-z_{j}^{2}\right) \prod_{1 \leq j<k \leq N}\left(1-z_{j} z_{k}\right)\left(-1+z_{j} z_{k}^{-1}\right)} \\
& \times \sum_{\sigma \in S_{N}} \sum_{\tau_{1}= \pm 1, \ldots, \tau_{N}= \pm 1}(-1)^{\sigma}(-1)^{|\tau|} \prod_{j=1}^{N} \prod_{k=0}^{x_{j}-1}\left\{\alpha_{k}+\left(1-\alpha_{k} \gamma_{k}\right) z_{\sigma(j)}^{\tau_{\sigma(j)}}\right\} \\
& \times \prod_{j=1}^{N} \prod_{k=x_{j}+1}^{M-1}\left(1-\gamma_{k} z_{\sigma(j)}^{\tau_{\sigma(j)}}\right) \prod_{j=1}^{N} \prod_{k=1}^{M-1}\left(1-\gamma_{k} z_{\sigma(j)}^{-\tau_{\sigma(j)}}\right) \prod_{j=1}^{N}\left(1-\gamma_{M} z_{j}\right) \prod_{j=1}^{N}\left(1-\gamma_{M} z_{j}^{-1}\right) \\
= & \prod_{j=1}^{N}\left(1-\gamma_{M} z_{j}\right) \prod_{j=1}^{N}\left(1-\gamma_{M} z_{j}^{-1}\right) F_{M-1, N}^{\mathrm{I}}\left(z_{1}, \ldots, z_{N}\left|\gamma_{1}, \ldots, \gamma_{M-1}\right| x_{1}, \ldots, x_{N}\right), \quad(4.28)
\end{aligned}
$$

which shows that $F_{M, N}\left(z_{1}, \ldots, z_{N}\left|\gamma_{1}, \ldots, \gamma_{M}\right| x_{1}, \ldots, x_{N}\right)$ satisfies Property (3) for the case $x_{N} \neq M$.

\section{Dual Cauchy formula for generalized symplectic Schur func- tions}

In this section, as an application of the correspondence between the type I wavefunctions and the generalized symplectic Schur functions, we derive the dual Cauchy formula for the 
generalized symplectic Schur functions. We apply the idea due to Bump-McNamara-Nakasuji [12], which they derived the dual Cauchy formula for factorial Schur functions by evaluating the domain wall boundary partition functions in two ways and comparing the two evaluations. The domain wall boundary partition functions $Z_{M}^{\mathrm{I}}\left(z_{1}, \ldots, z_{M} \mid \gamma_{1}, \ldots, \gamma_{M}\right)$ are special cases $M=N, x_{j}=j, j=1, \ldots, M$ of the wavefunctions

$$
Z_{M}^{\mathrm{I}}\left(z_{1}, \ldots, z_{M} \mid \gamma_{1}, \ldots, \gamma_{M}\right):=\Phi_{M, M}^{\mathrm{I}}\left(z_{1}, \ldots, z_{M}\left|\gamma_{1}, \ldots, \gamma_{M}\right| 1, \ldots, M\right) .
$$

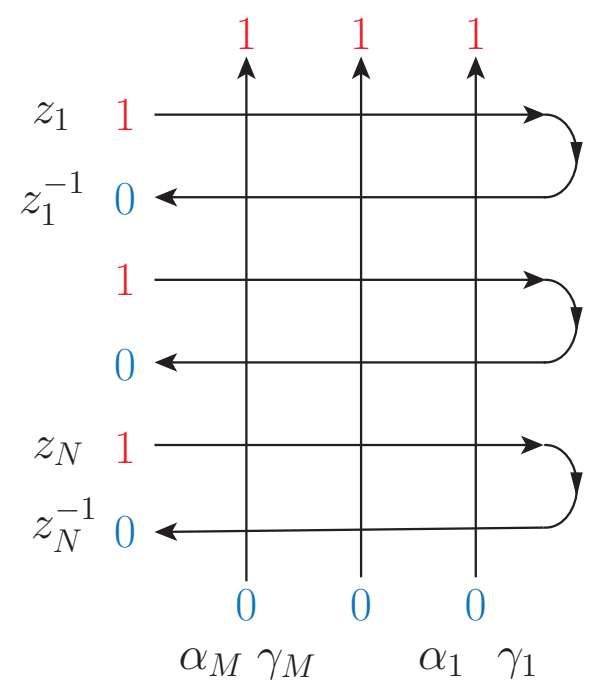

Figure 11: The domain wall boundary partition functions $Z_{M}^{\mathrm{I}}\left(z_{1}, \ldots, z_{M} \mid \gamma_{1}, \ldots, \gamma_{M}\right)$ (5.2), $Z_{M}^{\mathrm{II}}\left(z_{1}, \ldots, z_{M} \mid \gamma_{1}, \ldots, \gamma_{M}\right)$ (7.2) under reflecting boundary.

First, one can show the following factorization formula for the type I domain wall boundary partition functions.

Theorem 5.1. The type I domain wall boundary partition functions $Z_{M}^{\mathrm{I}}\left(z_{1}, \ldots, z_{M} \mid \gamma_{1}, \ldots, \gamma_{M}\right)$ have the following factorized form:

$$
\begin{aligned}
& Z_{M}^{\mathrm{I}}\left(z_{1}, \ldots, z_{M} \mid \gamma_{1}, \ldots, \gamma_{M}\right)=\prod_{j=1}^{M} z_{j}^{j-1-M}\left(1+t z_{j}^{2}\right) \prod_{1 \leq j<k \leq M}\left(1+t z_{j} z_{k}\right)\left(1+t z_{j} z_{k}^{-1}\right) \\
& \quad \times \prod_{0 \leq j<k \leq M}\left\{1+\alpha_{j}\left(\gamma_{k}-\gamma_{j}\right)\right\} \prod_{1 \leq j<k \leq M}\left(1-\gamma_{j} \gamma_{k}\right) .
\end{aligned}
$$

Proof. Since the type I domain wall boundary partition functions $Z_{M}^{\mathrm{I}}\left(z_{1}, \ldots, z_{M} \mid \gamma_{1}, \ldots, \gamma_{M}\right)$ are special cases of the type I wavefunctions $\Phi_{M, M}^{\mathrm{I}}\left(z_{1}, \ldots, z_{M}\left|\gamma_{1}, \ldots, \gamma_{M}\right| 1, \ldots, M\right)$ (5.1), what we need is to prove the following special case of Proposition 4.4 .

Proposition 5.2. The type I domain wall boundary partition functions $Z_{M}^{\mathrm{I}}\left(z_{1}, \ldots, z_{M} \mid \gamma_{1}, \ldots, \gamma_{M}\right)$ satisfies the following properties. 
(1) The type I domain wall boundary partition functions $Z_{M}^{\mathrm{I}}\left(z_{1}, \ldots, z_{M} \mid \gamma_{1}, \ldots, \gamma_{M}\right)$ is a polynomial of degree $2 M-1$ in $\gamma_{M}$.

(2) The following form

$$
\frac{Z_{M}^{\mathrm{I}}\left(z_{1}, \ldots, z_{M} \mid \gamma_{1}, \ldots, \gamma_{M}\right)}{\prod_{j=1}^{M} z_{j}^{j-1-M}\left(1+t z_{j}^{2}\right) \prod_{1 \leq j<k \leq M}\left(1+t z_{j} z_{k}\right)\left(1+t z_{j} z_{k}^{-1}\right)},
$$

is symmetric with respect to $z_{1}, \ldots, z_{M}$, and is invariant under the exchange $z_{i} \longleftrightarrow z_{i}^{-1}$ for $i=1, \ldots, M$.

(3) The following recursive relations between the type I domain wall boundary partition functions hold:

$$
\begin{aligned}
& \left.Z_{M}^{\mathrm{I}}\left(z_{1}, \ldots, z_{M} \mid \gamma_{1}, \ldots, \gamma_{M}\right)\right|_{\gamma_{M}=z_{M}} \\
& \quad=\prod_{j=1}^{M}\left(t z_{M} z_{j}+1\right) \prod_{j=1}^{M-1}\left(t+z_{M} z_{j}^{-1}\right) \prod_{j=0}^{M-1}\left\{\left(1-\alpha_{j} \gamma_{j}\right) z_{M}^{-1}+\alpha_{j}\right\} \prod_{j=1}^{M-1}\left(1-\gamma_{j} z_{M}\right) \\
& \quad \times Z_{M-1}^{\mathrm{I}}\left(z_{1}, \ldots, z_{M-1} \mid \gamma_{1}, \ldots, \gamma_{M-1}\right) .
\end{aligned}
$$

(4) The following holds for the case $M=1$ :

$$
Z_{1}^{\mathrm{I}}\left(z \mid \gamma_{1}\right)=\frac{1+t z^{2}}{z^{2}-1} \sum_{\tau= \pm 1} \tau\left\{\alpha_{0}+\left(1-\alpha_{0} \gamma_{0}\right) z^{\tau}\right\}\left(1-\gamma_{1} z^{-\tau}\right) .
$$

It is easy to see that the right hand side of $(5.2)$ satisfies all the properties listed in Proposition 5.2 .

Now we derive the dual Cauchy formula for the generalized symplectic Schur functions.

Theorem 5.3. The following dual Cauchy formula holds for the generalized symplectic Schur functions with sets variables $\{x\}_{N}=\left\{x_{1}, \ldots, x_{N}\right\},\{y\}_{M}=\left\{y_{1}, \ldots, y_{M}\right\},\{\bar{\alpha}\}=$ $\left\{\alpha_{0}, \ldots, \alpha_{N+M}\right\},\{\bar{\gamma}\}=\left\{\gamma_{0}, \ldots, \gamma_{N+M}\right\}$,

$$
\begin{aligned}
& \sum_{\lambda \subseteq M^{N}} s p_{\lambda}\left(\{x\}_{N}|\{\bar{\alpha}\}|\{\bar{\gamma}\}\right) s p_{\hat{\lambda}}\left(\{y\}_{M}|\{-\bar{\alpha}\}|\{-\bar{\gamma}\}\right) \\
= & \prod_{j=1}^{M} y_{j}^{-N} \prod_{j=1}^{N} \prod_{k=1}^{M}\left(1+x_{j} y_{k}\right)\left(1+x_{j}^{-1} y_{k}\right) \prod_{0 \leq j<k \leq N+M}\left(1+\alpha_{j}\left(\gamma_{k}-\gamma_{j}\right)\right) \prod_{1 \leq j<k \leq N+M}\left(1-\gamma_{j} \gamma_{k}\right),
\end{aligned}
$$

where $\{-\bar{\alpha}\}=\left\{-\alpha_{0}, \ldots,-\alpha_{N+M}\right\},\{-\bar{\gamma}\}=\left\{-\gamma_{0}, \ldots,-\gamma_{N+M}\right\}$ and $\hat{\lambda}=\left(\hat{\lambda}_{1}, \ldots, \hat{\lambda}_{M}\right)$ is the partition of the Young diagram $\lambda=\left(\lambda_{1}, \ldots, \lambda_{N}\right)$ given by

$$
\hat{\lambda}_{i}=\left|\left\{j \mid \lambda_{j} \leq M-i\right\}\right| \text {. }
$$

Proof. First, we have shown Theorem 5.1 which states that the type I domain wall boundary partition functions $Z_{M}^{\mathrm{I}}\left(z_{1}, \ldots, z_{M} \mid \gamma_{1}, \ldots, \gamma_{M}\right)$ have the factorized form (5.2). 
On the other hand, one can evaluate the domain wall boundary partition functions by inserting the completeness relation

$$
\sum_{\{x\}}\left|x_{1} \cdots x_{N}\right\rangle\left\langle x_{1} \cdots x_{N}\right|=\mathrm{Id},
$$

between the double-row $B$-operators and using the correspondences between the wavefunctions, the dual wavefunctions and the generalized symplectic Schur functions 4.18) and 4.19) to get

$$
\begin{aligned}
Z_{M}^{\mathrm{I}}( & \left.z_{1}, \ldots, z_{M} \mid \gamma_{1}, \ldots, \gamma_{M}\right) \\
= & \left\langle 1^{M}\left|\mathcal{B}^{\mathrm{I}}\left(z_{1},\{\bar{\alpha}\},\{\bar{\gamma}\}\right) \cdots \mathcal{B}^{\mathrm{I}}\left(z_{M},\{\bar{\alpha}\},\{\bar{\gamma}\}\right)\right| 0^{M}\right\rangle \\
= & \sum_{\{x\}}\left\langle 1^{M}\left|\mathcal{B}^{\mathrm{I}}\left(z_{1},\{\bar{\alpha}\},\{\bar{\gamma}\}\right) \cdots \mathcal{B}^{\mathrm{I}}\left(z_{M-N},\{\bar{\alpha}\},\{\bar{\gamma}\}\right)\right| x_{1} \cdots x_{N}\right\rangle \\
& \times\left\langle x_{1} \cdots x_{N}\left|\mathcal{B}^{\mathrm{I}}\left(z_{M-N+1},\{\bar{\alpha}\},\{\bar{\gamma}\}\right) \cdots \mathcal{B}^{\mathrm{I}}\left(z_{M},\{\bar{\alpha}\},\{\bar{\gamma}\}\right)\right| 0^{M}\right\rangle \\
= & \sum_{x \sqcup \bar{x}=\{1, \ldots, M\}}\left\langle 1^{M}\left|\mathcal{B}^{\mathrm{I}}\left(z_{1},\{\bar{\alpha}\},\{\bar{\gamma}\}\right) \cdots \mathcal{B}^{\mathrm{I}}\left(z_{M-N},\{\bar{\alpha}\},\{\bar{\gamma}\}\right)\right| \overline{x_{1}} \cdots \overline{x_{M-N}}\right\rangle \\
& \times\left\langle x_{1} \cdots x_{N}\left|\mathcal{B}^{\mathrm{I}}\left(z_{M-N+1},\{\bar{\alpha}\},\{\bar{\gamma}\}\right) \cdots \mathcal{B}^{\mathrm{I}}\left(z_{M-N},\{\bar{\alpha}\},\{\bar{\gamma}\}\right)\right| 0^{M}\right\rangle \\
= & \sum_{x \sqcup \bar{x}=\{1,2, \ldots, M\}} \bar{\Phi}_{M, M-N}^{\mathrm{I}}\left(z_{1}, \ldots, z_{M-N}\left|\gamma_{1}, \ldots, \gamma_{M}\right| \overline{x_{1}}, \ldots, \overline{x_{M-N}}\right) \\
& \times \Phi_{M, N}^{\mathrm{I}}\left(z_{M-N+1}, \ldots, z_{M}\left|\gamma_{1}, \ldots, \gamma_{M}\right| x_{1}, \ldots, x_{N}\right) \\
= & \sum_{\lambda \subseteq(M-N)^{N}} t^{N(M-N)} \prod_{j=1}^{M-N} z_{j}^{j-1-M+N}\left(1+t z_{j}^{2}\right) \prod_{1 \leq j<k \leq M-N}\left(1+t z_{j} z_{k}\right)\left(1+t z_{j} z_{k}^{-1}\right) \\
& \times s p_{\bar{\lambda}}\left(t z_{1}, \ldots, t z_{M-N}|\{-\bar{\alpha}\}|\{-\bar{\gamma}\}\right) \\
& \times \prod_{j=M-N+1}^{M} z_{j}^{j-1-M}\left(1+t z_{j}^{2}\right) \\
& \times s p_{\lambda}\left(z_{M-N+1}, \ldots, z_{M}|\{\bar{\alpha}\}|\{\bar{\gamma}\}\right) .
\end{aligned}
$$

Note that the sum over all particle configurations $\{x\}$ is translated to the sum over all Young diagrams $\lambda$ satisfying $\lambda \subseteq(M-N)^{N}$. Comparing the two ways of evaluations (5.9) and (5.2) and cancelling common factors, we get the following identity

$$
\begin{aligned}
& \prod_{j=1}^{M-N}\left(t z_{j}\right)^{-N} \prod_{\substack{1 \leq j \leq M-N \\
M-N+1 \leq k \leq M}}\left(1+t z_{j} z_{k}\right)\left(1+t z_{j} z_{k}^{-1}\right) \\
& \quad \times \prod_{0 \leq j<k \leq M}\left(1+\alpha_{j}\left(\gamma_{k}-\gamma_{j}\right)\right) \prod_{1 \leq j<k \leq M}\left(1-\gamma_{j} \gamma_{k}\right) \\
& \quad=\sum_{\lambda \subseteq(M-N)^{N}} s p_{\bar{\lambda}}\left(t z_{1}, \ldots, t z_{M-N}|\{-\bar{\alpha}\}|\{-\bar{\gamma}\}\right) s p_{\lambda}\left(z_{M-N+1}, \ldots, z_{M}|\{\bar{\alpha}\}|\{\bar{\gamma}\}\right),
\end{aligned}
$$

which, after some reparametrization, can be rewritten in the form 5.6).

The dual Cauchy formula (5.6) is a generalization of the one for the ordinary symplectic Schur functions which were proven by various ways $[78,79,80,81,82,83,84$. 


\section{Type II wavefunctions and generalized Bump-Friedberg- Hoffstein Whittaker functions}

In this and the next sections, we perform the analysis on the type II wavefunctions. The arguments, statements and the proofs are similar to those for the type I wavefunctions.

\subsection{One particle case}

The simplest case $N=1$ of type II wavefunctions can be calculated using the identity 4.1 in Lemma 4.1 as well.

Proposition 6.1. The type II wavefunction $\Phi_{M, 1}^{\mathrm{II}}\left(z\left|\gamma_{1}, \ldots, \gamma_{M}\right| x_{1}\right)$ is explicitly expressed as

$$
\begin{aligned}
& \Phi_{M, 1}^{\mathrm{II}}\left(z\left|\gamma_{1}, \ldots, \gamma_{M}\right| x_{1}\right)=\frac{z^{1 / 2}(1-\sqrt{-t} z)}{z^{2}-1} \\
& \quad \times \sum_{\tau= \pm 1} \tau\left(z^{\tau}+\sqrt{-t}\right) \prod_{j=1}^{x_{1}-1}\left(\alpha_{j}+\left(1-\alpha_{j} \gamma_{j}\right) z^{\tau}\right) \prod_{j=x_{1}+1}^{M}\left(1-\gamma_{j} z^{\tau}\right) \prod_{j=1}^{M}\left(1-\gamma_{j} z^{-\tau}\right) .
\end{aligned}
$$

Proof. We decompose $\Phi_{M, 1}^{\mathrm{II}}\left(z\left|\gamma_{1}, \ldots, \gamma_{M}\right| x_{1}\right)$ as

$$
\begin{aligned}
\Phi_{M, 1}^{\mathrm{II}}(z \mid & \left.\gamma_{1}, \ldots, \gamma_{M} \mid x_{1}\right) \\
= & -\sqrt{-t} z^{1 / 2}\left\langle x_{1}|\widetilde{B}(z,\{\alpha\},\{\gamma\})| 0^{M}\right\rangle\left\langle 0^{M}|A(z,\{\alpha\},\{\gamma\})| 0^{M}\right\rangle \\
& +z^{-1 / 2}\left\langle x_{1}|\widetilde{A}(z,\{\alpha\},\{\gamma\})| x_{1}\right\rangle\left\langle x_{1}|B(z,\{\alpha\},\{\gamma\})| 0^{M}\right\rangle \\
& +z^{-1 / 2} \sum_{j=1}^{x_{1}-1}\left\langle x_{1}|\widetilde{A}(z,\{\alpha\},\{\gamma\})| j\right\rangle\left\langle j|B(z,\{\alpha\},\{\gamma\})| 0^{M}\right\rangle,
\end{aligned}
$$

to get

$$
\begin{aligned}
\Phi_{M, 1}^{\mathrm{II}}\left(z\left|\gamma_{1}, \ldots, \gamma_{M}\right| x_{1}\right)=\prod_{j=x_{1}+1}^{M}\left(1-\gamma_{j} z\right)\left(1-\gamma_{j} z^{-1}\right) \\
\times\left[-\sqrt{-t} z^{1 / 2} \prod_{k=1}^{x_{1}-1}\left\{\left(1-\alpha_{k} \gamma_{k}\right) z+\alpha_{k}\right\} \prod_{k=1}^{x_{1}}\left(1-\gamma_{k} z^{-1}\right)\right. \\
+z^{-1 / 2}\left(t \gamma_{x_{1}} z+1\right) \prod_{k=1}^{x_{1}-1}\left\{\left(1-\alpha_{k} \gamma_{k}\right) z^{-1}+\alpha_{k}\right\}\left(1-\gamma_{k} z\right) \\
+(t+1) z^{1 / 2} \sum_{j=1}^{x_{1}-1} \prod_{k=1}^{j-1}\left\{\left(1-\alpha_{k} \gamma_{k}\right) z^{-1}+\alpha_{k}\right\}\left(1-\gamma_{k} z\right) \\
\left.\quad \times \prod_{k=j+1}^{x_{1}-1}\left\{\left(1-\alpha_{k} \gamma_{k}\right) z+\alpha_{k}\right\} \prod_{k=j+1}^{x_{1}}\left(1-\gamma_{k} z^{-1}\right)\right]
\end{aligned}
$$

which, by using the equality (4.1) in Lemma 4.1, can be rewritten into the form (6.1).

We call similarly calculate type II dual wavefunction $\bar{\Phi}_{M, 1}^{\mathrm{II}}\left(z\left|\gamma_{1}, \ldots, \gamma_{M}\right| \overline{x_{1}}\right)$. 
Proposition 6.2. The type II dual wavefunction $\bar{\Phi}_{M, 1}^{\mathrm{II}}\left(z\left|\gamma_{1}, \ldots, \gamma_{M}\right| \overline{x_{1}}\right)$ is explicitly expressed as

$$
\begin{aligned}
\bar{\Phi}_{M, 1}^{\mathrm{II}}\left(z\left|\gamma_{1}, \ldots, \gamma_{M}\right| \overline{x_{1}}\right) \\
=\frac{t^{M} z^{1 / 2}(1-\sqrt{-t} z)}{t^{2} z^{2}-1} \sum_{\tau= \pm 1} \tau\left\{(t z)^{\tau}-\sqrt{-t}\right\} \prod_{j=1}^{\overline{x_{1}}-1}\left\{-\alpha_{j}+\left(1-\alpha_{j} \gamma_{j}\right)(t z)^{\tau}\right\} \\
\quad \times \prod_{j=\overline{x_{1}}+1}^{M}\left\{1+\gamma_{j}(t z)^{\tau}\right\} \prod_{j=1}^{M}\left\{1+\gamma_{j}(t z)^{-\tau}\right\} .
\end{aligned}
$$

\subsection{Izergin-Korepin analysis}

We can use the Izergin-Korepin technique [6, 7, 29] to extract the properties for the type II wavefunctions as well.

Proposition 6.3. The type II wavefunctions $\Phi_{M, N}^{\mathrm{II}}\left(z_{1}, \ldots, z_{N}\left|\gamma_{1}, \ldots, \gamma_{M}\right| x_{1}, \ldots, x_{N}\right)$ satisfies the following properties.

(1) When $x_{N}=M$, the type II wavefunctions $\Phi_{M, N}^{\mathrm{II}}\left(z_{1}, \ldots, z_{N}\left|\gamma_{1}, \ldots, \gamma_{M}\right| x_{1}, \ldots, x_{N}\right)$ is a polynomial of degree $2 N-1$ in $\gamma_{M}$.

(2) The following form

$$
\frac{\Phi_{M, N}^{\mathrm{II}}\left(z_{1}, \ldots, z_{N}\left|\gamma_{1}, \ldots, \gamma_{M}\right| x_{1}, \ldots, x_{N}\right)}{\prod_{j=1}^{N} z_{j}^{j-1 / 2-N}\left(1-\sqrt{-t} z_{j}\right) \prod_{1 \leq j<k \leq N}\left(1+t z_{j} z_{k}\right)\left(1+t z_{j} z_{k}^{-1}\right)},
$$

is symmetric with respect to $z_{1}, \ldots, z_{N}$, and is invariant under the exchange $z_{i} \longleftrightarrow z_{i}^{-1}$ for $i=1, \ldots, N$.

(3) The following recursive relations between the type II wavefunctions hold if $x_{N}=M$ :

$$
\begin{aligned}
& \left.\Phi_{M, N}^{\mathrm{II}}\left(z_{1}, \ldots, z_{N}\left|\gamma_{1}, \ldots, \gamma_{M}\right| x_{1}, \ldots, x_{N}\right)\right|_{\gamma_{M}=z_{N}} \\
& =z_{N}^{-1 / 2} \prod_{j=1}^{N}\left(t z_{N} z_{j}+1\right) \prod_{j=1}^{N-1}\left(t+z_{N} z_{j}^{-1}\right) \prod_{j=1}^{M-1}\left\{\left(1-\alpha_{j} \gamma_{j}\right) z_{N}^{-1}+\alpha_{j}\right\} \prod_{j=1}^{M-1}\left(1-\gamma_{j} z_{N}\right) \\
& \quad \times \Phi_{M-1, N-1}^{\mathrm{I}}\left(z_{1}, \ldots, z_{N-1}\left|\gamma_{1}, \ldots, \gamma_{M-1}\right| x_{1}, \ldots, x_{N-1}\right) .
\end{aligned}
$$

If $x_{N} \neq M$, the following factorizations hold for the type II wavefunctions:

$$
\begin{aligned}
& \Phi_{M, N}^{\mathrm{II}}\left(z_{1}, \ldots, z_{N}\left|\gamma_{1}, \ldots, \gamma_{M}\right| x_{1}, \ldots, x_{N}\right) \\
& \quad=\prod_{j=1}^{N}\left(1-\gamma_{M} z_{j}\right)\left(1-\gamma_{M} z_{j}^{-1}\right) \Phi_{M-1, N}^{\mathrm{II}}\left(z_{1}, \ldots, z_{N}\left|\gamma_{1}, \ldots, \gamma_{M-1}\right| x_{1}, \ldots, x_{N}\right) .
\end{aligned}
$$

(4) The following holds for the case $N=1, x_{1}=M$ :

$$
\begin{aligned}
& \Phi_{M, 1}^{\mathrm{II}}\left(z\left|\gamma_{1}, \ldots, \gamma_{M}\right| M\right) \\
& \quad=\frac{z^{1 / 2}(1-\sqrt{-t} z)}{z^{2}-1} \sum_{\tau= \pm 1} \tau\left(z^{\tau}+\sqrt{-t}\right) \prod_{j=1}^{M-1}\left\{\alpha_{j}+\left(1-\alpha_{j} \gamma_{j}\right) z^{\tau}\right\} \prod_{j=1}^{M}\left(1-\gamma_{j} z^{-\tau}\right) .
\end{aligned}
$$


Proof. Proposition 6.3 can be proved in the same way with Proposition 4.4 .

Property (1) and (6.7) in Property (3) is the same with the corresponding ones in Proposition 4.4, since the difference of the $K$-matrices one uses for the type I and type II wavefunctions do not affect the argument to show these properties.

Property (2) can be proved in the same way with Brubaker-Bump-Chinta-Gunnells [46], which they used the arguments by Ivanov [43, 44] based on the caduceus relations and the fish relations (see Appendix A for details). The denominator in $(6.5)$ is different from that in (4.6). This difference comes from the fact that for type II wavefunctions, we use the $K$-matrix (3.5) instead of (3.3) at the boundary.

The difference of the $K$-matrices are also reflected in the difference between $(6.6)$ in Property (3) and Property (4) in Proposition 4.4 and Proposition 6.3

We also list below the properties for the type II dual wavefunctions.

Proposition 6.4. The type II dual wavefunctions $\bar{\Phi}_{M, N}^{\mathrm{II}}\left(z_{1}, \ldots, z_{N}\left|\gamma_{1}, \ldots, \gamma_{M}\right| \overline{x_{1}}, \ldots, \overline{x_{N}}\right)$ satisfies the following properties.

(1) When $\overline{x_{N}}=M$, the type II dual wavefunctions $\bar{\Phi}_{M, N}^{\mathrm{II}}\left(z_{1}, \ldots, z_{N}\left|\gamma_{1}, \ldots, \gamma_{M}\right| \overline{x_{1}}, \ldots, \overline{x_{N}}\right)$ is a polynomial of degree $2 N-1$ in $\gamma_{M}$.

(2) The following form

$$
\frac{\bar{\Phi}_{M, N}^{\mathrm{II}}\left(z_{1}, \ldots, z_{N}\left|\gamma_{1}, \ldots, \gamma_{M}\right| x_{1}, \ldots, x_{N}\right)}{\prod_{j=1}^{N} z_{j}^{j-1 / 2-N}\left(1-\sqrt{-t} z_{j}\right) \prod_{1 \leq j<k \leq N}\left(1+t z_{j} z_{k}\right)\left(1+t z_{j} z_{k}^{-1}\right)},
$$

is symmetric with respect to $z_{1}, \ldots, z_{N}$, and is invariant under the exchange $z_{i} \longleftrightarrow z_{i}^{-1}$ for $i=1, \ldots, N$.

(3) The following recursive relations between the type II dual wavefunctions hold if $\overline{x_{N}}=M$ :

$$
\begin{aligned}
& \left.\bar{\Phi}_{M, N}^{\mathrm{II}}\left(z_{N}, \ldots, z_{1}\left|\gamma_{1}, \ldots, \gamma_{M}\right| \overline{x_{1}}, \ldots, \overline{x_{N}}\right)\right|_{\gamma_{M}=-t^{-1} z_{N}^{-1}} \\
= & -\sqrt{-t} z_{N}^{1 / 2} \prod_{j=1}^{N}\left(1+\frac{1}{t z_{N} z_{j}}\right) \prod_{j=1}^{N-1}\left(1+\frac{z_{j}}{t z_{N}}\right) \prod_{j=1}^{M-1}\left(t\left(1-\alpha_{j} \gamma_{j}\right) z_{N}-\alpha_{j}\right) \prod_{j=1}^{M-1}\left(t+\gamma_{j} z_{N}^{-1}\right) \\
& \times \bar{\Phi}_{M-1, N-1}^{\mathrm{II}}\left(z_{N-1}, \ldots, z_{1}\left|\gamma_{1}, \ldots, \gamma_{M-1}\right| \overline{x_{1}}, \ldots, \overline{x_{N-1}}\right) .
\end{aligned}
$$

If $\overline{x_{N}} \neq M$, the following factorizations hold for the type II dual wavefunctions:

$$
\begin{aligned}
& \bar{\Phi}_{M, N}^{\mathrm{II}}\left(z_{1}, \ldots, z_{N}\left|\gamma_{1}, \ldots, \gamma_{M}\right| \overline{x_{1}}, \ldots, \overline{x_{N}}\right) \\
= & \prod_{j=1}^{N}\left(1+t \gamma_{M} z_{j}\right)\left(t+\gamma_{M} z_{j}^{-1}\right) \bar{\Phi}_{M-1, N}^{\mathrm{II}}\left(z_{1}, \ldots, z_{N}\left|\gamma_{1}, \ldots, \gamma_{M-1}\right| \overline{x_{1}}, \ldots, \overline{x_{N}}\right) .
\end{aligned}
$$


(4) The following holds for the case $N=1, \overline{x_{1}}=M$ :

$$
\begin{aligned}
& \bar{\Phi}_{M, 1}^{\mathrm{II}}\left(z\left|\gamma_{1}, \ldots, \gamma_{M}\right| M\right) \\
= & \frac{t^{M} z^{1 / 2}(1-\sqrt{-t} z)}{t^{2} z^{2}-1} \sum_{\tau= \pm 1} \tau\left((t z)^{\tau}-\sqrt{-t}\right) \\
& \times \prod_{j=1}^{M-1}\left(-\alpha_{j}+\left(1-\alpha_{j} \gamma_{j}\right)(t z)^{\tau}\right) \prod_{j=1}^{M}\left(1+\gamma_{j}(t z)^{-\tau}\right) .
\end{aligned}
$$

\subsection{Generalized Bump-Friedberg-Hoffstein Whittaker functions}

We first introduce the following symmetric functions which generalizes the Whittaker functions introduced by Bump, Friedberg and Hoffstein [60].

Definition 6.5. We define two generalized Bump-Friedberg-Hoffstein Whittaker functions to be the following determinants:

$$
o_{\lambda}^{ \pm}\left(\{z\}_{N}|\{\alpha\}|\{\gamma\} \mid t\right)=\frac{H_{\lambda+\delta}^{ \pm}\left(\{z\}_{N}|\{\alpha\}|\{\gamma\} \mid t\right)}{\operatorname{det}_{N}\left(z_{k}^{N-j+1}-z_{k}^{-N+j-1}\right)} .
$$

Here, $\{z\}_{N}=\left\{z_{1}, \ldots, z_{N}\right\}$ is a set of symmetric variables, $\{\alpha\}$ and $\{\gamma\}$ are sets of variables $\{\alpha\}=\left\{\alpha_{1}, \ldots, \alpha_{M}\right\}$ and $\{\gamma\}=\left\{\gamma_{1}, \ldots, \gamma_{M}\right\}, \lambda$ denotes a Young diagram $\lambda=$ $\left(\lambda_{1}, \lambda_{2}, \ldots, \lambda_{N}\right)$ with weakly decreasing non-negative integers $\lambda_{1} \geq \lambda_{2} \geq \cdots \geq \lambda_{N} \geq 0$, and $\delta=(N-1, N-2, \ldots, 0) . H_{\mu}^{ \pm}\left(\{z\}_{N}|\{\alpha\}|\{\gamma\} \mid t\right)$ are $N \times N$ determinants

$$
H_{\mu}^{ \pm}\left(\{z\}_{N}|\{\alpha\}|\{\gamma\} \mid t\right)=\operatorname{det}_{N}\left(h_{\mu_{j}}^{ \pm}\left(z_{k}|\{\alpha\}|\{\gamma\} \mid t\right)-h_{\mu_{j}}^{ \pm}\left(z_{k}^{-1}|\{\alpha\}|\{\gamma\} \mid t\right)\right),
$$

where

$$
h_{\mu}^{ \pm}(z|\{\alpha\}|\{\gamma\} \mid t)=(z \pm \sqrt{-t}) \prod_{j=1}^{\mu}\left(\alpha_{j}+\left(1-\alpha_{j} \gamma_{j}\right) z\right) \prod_{j=\mu+2}^{M}\left(1-\gamma_{j} z\right) \prod_{j=1}^{M}\left(1-\gamma_{j} z^{-1}\right) .
$$

The generalized Whittaker functions (6.13) are symmetric with respect to the variables $z_{1}, \ldots, z_{N}$, and are invariant under the exchange $z_{i} \longleftrightarrow z_{i}^{-1}$ for $i=1, \ldots, N$. If one sets $\alpha_{j}=\gamma_{j}=0(j=1, \ldots, M)$, the generalized Whittaker functions reduce to the Whittaker functions introduced by Bump, Friedberg and Hoffstein [60].

Theorem 6.6. The type II wavefunctions $\Phi_{M, N}^{\mathrm{II}}\left(z_{1}, \ldots, z_{N}\left|\gamma_{1}, \ldots, \gamma_{M}\right| x_{1}, \ldots, x_{N}\right)$ are explicitly expressed using the generalized Bump-Friedberg-Hoffstein Whittaker functions as

$$
\begin{aligned}
& \Phi_{M, N}^{\mathrm{II}}\left(z_{1}, \ldots, z_{N}\left|\gamma_{1}, \ldots, \gamma_{M}\right| x_{1}, \ldots, x_{N}\right) \\
= & \prod_{j=1}^{N} z_{j}^{j-1 / 2-N}\left(1-\sqrt{-t} z_{j}\right) \prod_{1 \leq j<k \leq N}\left(1+t z_{j} z_{k}\right)\left(1+t z_{j} z_{k}^{-1}\right) o_{\lambda}^{+}\left(\{z\}_{N}|\{\alpha\}|\{\gamma\} \mid t\right),
\end{aligned}
$$

under the relation $\lambda_{j}=x_{N-j+1}-N+j-1, j=1, \ldots, N$. 
The type II dual wavefunctions $\bar{\Phi}_{M, N}^{\mathrm{II}}\left(z_{1}, \ldots, z_{N}\left|\gamma_{1}, \ldots, \gamma_{M}\right| \overline{x_{1}}, \ldots, \overline{x_{N}}\right)$ are explicitly expressed using the generalized Bump-Friedberg-Hoffstein Whittaker functions as

$$
\begin{aligned}
& \bar{\Phi}_{M, N}^{\mathrm{II}}\left(z_{1}, \ldots, z_{N}\left|\gamma_{1}, \ldots, \gamma_{M}\right| \overline{x_{1}}, \ldots, \overline{x_{N}}\right) \\
= & t^{N(M-N)} \prod_{j=1}^{N} z_{j}^{j-1 / 2-N}\left(1-\sqrt{-t} z_{j}\right) \prod_{1 \leq j<k \leq N}\left(1+t z_{j} z_{k}\right)\left(1+t z_{j} z_{k}^{-1}\right) o_{\bar{\lambda}}^{-}\left(\{t z\}_{N}|\{-\alpha\}|\{-\gamma\} \mid t\right),
\end{aligned}
$$

under the relation $\overline{\lambda_{j}}=\overline{x_{N-j+1}}-N+j-1, j=1, \ldots, N$, and the symmetric variables are $\{t z\}_{N}=\left\{t z_{1}, \ldots, t z_{N}\right\}$. Moreover, the signs of the parameters of the generalized symplectic Schur functions in the right hand side of (4.19) are now inverted simultaneously: $\{-\alpha\}=$ $\left\{-\alpha_{1}, \ldots,-\alpha_{M}\right\}$ and $\{-\gamma\}=\left\{-\gamma_{1}, \ldots,-\gamma_{M}\right\}$.

The correspondence (6.16) is a generalization of the conjecture given in the work by Brubaker-Bump-Chinta-Gunnells [46] for the case $\alpha_{j}=\gamma_{j}=0, j=1, \ldots, M$, in which they conjectured the relation between the wavefunctions of type $B$ ice models and the Whittaker functions by Bump-Friedberg-Hoffstein [60].

Proof. The correspondence $(6.16)$ can be proved in the same way with proving (4.18). We show that the right hand side of $(6.16)$ satisfies all the Properties in Proposition 6.3.

Let us illustrate the proof of Property (3) for the case $x_{N}=M$, which is the hardest thing to check.

Again using 4.20 , the definition of the determinant and the correspondence between the positions of particles $\{x\}$ and the Young diagrams $\{\lambda\}$, we rewrite the right hand side of (6.16) as

$$
\begin{aligned}
F_{M, N}^{\mathrm{II}} & \left(z_{1}, \ldots, z_{N}\left|\gamma_{1}, \ldots, \gamma_{M}\right| x_{1}, \ldots, x_{N}\right) \\
:= & \frac{\prod_{j=1}^{N} z_{j}^{j-1 / 2-N}\left(1-\sqrt{-t} z_{j}\right) \prod_{1 \leq j<k \leq N}\left(1+t z_{j} z_{k}\right)\left(1+t z_{j} z_{k}^{-1}\right)}{(-1)^{N} \prod_{j=1}^{N} z_{j}^{j-1-N}\left(1-z_{j}^{2}\right) \prod_{1 \leq j<k \leq N}\left(1-z_{j} z_{k}\right)\left(-1+z_{j} z_{k}^{-1}\right)} \\
& \times \sum_{\sigma \in S_{N}} \sum_{\tau_{1}= \pm 1, \ldots, \tau_{N}= \pm 1}(-1)^{\sigma}(-1)^{|\tau|} \prod_{j=1}^{N}\left(z_{\sigma(j)}^{\tau_{\sigma(j)}}+\sqrt{-t}\right) \prod_{j=1}^{N} \prod_{k=1}^{x_{j}-1}\left(\alpha_{k}+\left(1-\alpha_{k} \gamma_{k}\right) z_{\sigma(j)}^{\tau_{\sigma(j)}}\right) \\
& \times \prod_{j=1}^{N} \prod_{k=x_{j}+1}^{M}\left(1-\gamma_{k} z_{\sigma(j)}^{\tau_{\sigma(j)}}\right) \prod_{j=1}^{N} \prod_{k=1}^{M}\left(1-\gamma_{k} z_{\sigma(j)}^{-\tau_{\sigma(j)}}\right),
\end{aligned}
$$

where $|\tau|$ denotes the number of $\tau_{j}$ s satisfying $\tau_{j}=-1$.

When $x_{N}=M$, only the summands satisfying $\sigma(N)=N, \tau_{N}=-1$ in (6.18) survive after specializing $\gamma_{M}$ to $\gamma_{M}=z_{N}$. Then we find that $\left.F_{M, N}^{\mathrm{II}}\left(z_{1}, \ldots, z_{N}\left|\gamma_{1}, \ldots, \gamma_{M}\right| x_{1}, \ldots, x_{N}\right)\right|_{\gamma_{M}=z_{N}}$ 
can be rewritten as

$$
\begin{aligned}
&\left.F_{M, N}^{\mathrm{II}}\left(z_{1}, \ldots, z_{N}\left|\gamma_{1}, \ldots, \gamma_{M}\right| x_{1}, \ldots, x_{N}\right)\right|_{\gamma_{M}=z_{N}} \\
&=-\frac{z_{N}^{1 / 2}\left(1-\sqrt{-t} z_{N}\right)}{1-z_{N}^{2}} \frac{\prod_{j=1}^{N-1}\left(1+t z_{j} z_{N}\right)\left(1+t z_{j} z_{N}^{-1}\right)}{\prod_{j=1}^{N-1}\left(1-z_{j} z_{N}\right)\left(-1+z_{j} z_{N}^{-1}\right)} \\
& \quad \times \frac{\prod_{j=1}^{N-1} z_{j}^{1 / 2}\left(1-\sqrt{-t} z_{j}\right) \prod_{1 \leq j<k \leq N-1}\left(1+t z_{j} z_{k}\right)\left(1+t z_{j} z_{k}^{-1}\right)}{(-1)^{N-1} \prod_{j=1}^{N-1}\left(1-z_{j}^{2}\right) \prod_{1 \leq j<k \leq N-1}\left(1-z_{j} z_{k}\right)\left(-1+z_{j} z_{k}^{-1}\right)} \\
& \quad \times \sum_{\sigma \in S_{N-1}} \sum_{1= \pm 1, \ldots, \tau_{N-1}= \pm 1}(-1)(-1)^{\sigma}(-1)^{|\tau|}\left(z_{N}^{-1}+\sqrt{-t}\right) \prod_{j=1}^{N-1}\left(z_{\sigma(j)}^{\tau_{\sigma(j)}}+\sqrt{-t}\right) \\
& \times \prod_{j=1}^{N-1} \prod_{k=1}^{x_{j}-1}\left\{\alpha_{k}+\left(1-\alpha_{k} \gamma_{k}\right) z_{\sigma(j)}^{\tau_{\sigma(j)}}\right\} \prod_{k=1}^{M-1}\left\{\alpha_{k}+\left(1-\alpha_{k} \gamma_{k}\right) z_{N}^{-1}\right\} \\
& \times \prod_{j=1}^{N-1} \prod_{k=x_{j}+1}^{M-1}\left(1-\gamma_{k} z_{\sigma(j)}^{\tau_{\sigma(j)}}\right) \prod_{j=1}^{N-1}\left(1-z_{N} z_{\sigma(j)}^{\tau_{\sigma(j)}}\right) \\
& \times\left(1-z_{N}^{2}\right) \prod_{k=1}^{M-1}\left(1-\gamma_{k} z_{N}\right) \prod_{j=1}^{N-1}\left(1-z_{N} z_{\sigma(j)}^{-\tau_{\sigma(j)}}\right) \prod_{j=1}^{N-1} \prod_{k=1}^{M-1}\left(1-\gamma_{k} z_{\sigma(j)}^{-\tau_{\sigma(j)}}\right) .
\end{aligned}
$$

We again use the obvious identity

$$
\prod_{j=1}^{N-1}\left(1-z_{N} z_{\sigma(j)}^{\tau_{\sigma(j)}}\right)\left(1-z_{N} z_{\sigma(j)}^{-\tau_{\sigma(j)}}\right)=\prod_{j=1}^{N-1}\left(1-z_{N} z_{j}\right)\left(1-z_{N} z_{j}^{-1}\right)
$$

and simplify (6.19) as

$$
\begin{aligned}
\left.F_{M, N}^{\mathrm{II}}\left(z_{1}, \ldots, z_{N}\left|\gamma_{1}, \ldots, \gamma_{M}\right| x_{1}, \ldots, x_{N}\right)\right|_{\gamma_{M}=z_{N}} \\
=z_{N}^{-1 / 2} \prod_{j=1}^{N}\left(t z_{N} z_{j}+1\right) \prod_{j=1}^{N-1}\left(t+z_{N} z_{j}^{-1}\right) \prod_{j=1}^{M-1}\left(\left(1-\alpha_{j} \gamma_{j}\right) z_{N}^{-1}+\alpha_{j}\right) \prod_{j=1}^{M-1}\left(1-\gamma_{j} z_{N}\right) \\
\quad \times \frac{\prod_{j=1}^{N-1} z_{j}^{j-1 / 2-(N-1)}\left(1-\sqrt{-t} z_{j}\right) \prod_{1 \leq j<k \leq N-1}\left(1+t z_{j} z_{k}\right)\left(1+t z_{j} z_{k}^{-1}\right)}{(-1)^{N-1} \prod_{j=1}^{N-1} z_{j}^{j-1-(N-1)}\left(1-z_{j}^{2}\right) \prod_{1 \leq j<k \leq N-1}\left(1-z_{j} z_{k}\right)\left(-1+z_{j} z_{k}^{-1}\right)} \\
\quad \times \sum_{\sigma \in S_{N-1} \tau_{1}= \pm 1, \ldots, \tau_{N-1}= \pm 1}(-1)^{\sigma}(-1)^{|\tau|} \prod_{j=1}^{N-1}\left(z_{\sigma(j)}^{\tau_{\sigma(j)}}+\sqrt{-t}\right) \\
\quad \times \prod_{j=1}^{N-1} \prod_{k=1}^{x_{j}-1}\left\{\alpha_{k}+\left(1-\alpha_{k} \gamma_{k}\right) z_{\sigma(j)}^{\tau_{\sigma(j)}}\right\} \prod_{j=1}^{N-1} \prod_{k=x_{j}+1}^{M-1}\left(1-\gamma_{k} z_{\sigma(j)}^{\tau_{\sigma(j)}}\right) \prod_{j=1}^{N-1} \prod_{k=1}^{M-1}\left(1-\gamma_{k} z_{\sigma(j)}^{-\tau_{\sigma(j)}}\right) \\
=z_{N}^{-1 / 2} \prod_{j=1}^{N}\left(t z_{N} z_{j}+1\right) \prod_{j=1}^{N-1}\left(t+z_{N} z_{j}^{-1}\right) \prod_{j=1}^{M-1}\left(\left(1-\alpha_{j} \gamma_{j}\right) z_{N}^{-1}+\alpha_{j}\right) \prod_{j=1}^{M-1}\left(1-\gamma_{j} z_{N}\right) \\
\quad \times F_{M-1, N-1}^{\mathrm{II}}\left(z_{1}, \ldots, z_{N-1}\left|\gamma_{1}, \ldots, \gamma_{M-1}\right| x_{1}, \ldots, x_{N-1}\right),
\end{aligned}
$$


hence we find that $F_{M, N}^{\mathrm{II}}\left(z_{1}, \ldots, z_{N}\left|\gamma_{1}, \ldots, \gamma_{M}\right| x_{1}, \ldots, x_{N}\right)$ satisfies Property (3) for the case $x_{N}=M$ in Proposition 6.3 .

\section{Dual Cauchy formula for generalized Bump-Friedberg-Hoffstein Whittaker functions}

We can derive the dual Cauchy formula for the generalized Whittaker functions in the same with deriving the one for the generalized symplectic Schur functions. We now deal the type II domain wall boundary partition functions.

$$
Z_{M}^{\mathrm{II}}\left(z_{1}, \ldots, z_{M} \mid \gamma_{1}, \ldots, \gamma_{M}\right)=\Phi_{M, M}^{\mathrm{II}}\left(z_{1}, \ldots, z_{M}\left|\gamma_{1}, \ldots, \gamma_{M}\right| 1, \ldots, M\right) .
$$

One again first shows the following factorization formula for the type II domain wall boundary partition functions.

Theorem 7.1. The type II domain wall boundary partition functions $Z_{M}^{\mathrm{II}}\left(z_{1}, \ldots, z_{M} \mid \gamma_{1}, \ldots, \gamma_{M}\right)$ have the following factorized form:

$$
\begin{aligned}
& Z_{M}^{\mathrm{II}}\left(z_{1}, \ldots, z_{M} \mid \gamma_{1}, \ldots, \gamma_{M}\right)=\prod_{j=1}^{M} z_{j}^{j-1 / 2-M}\left(1-\sqrt{-t} z_{j}\right)\left(1+\sqrt{-t} \gamma_{j}\right) \\
& \quad \times \prod_{1 \leq j<k \leq M}\left(1+t z_{j} z_{k}\right)\left(1+t z_{j} z_{k}^{-1}\right) \prod_{1 \leq j<k \leq M}\left\{1+\alpha_{j}\left(\gamma_{k}-\gamma_{j}\right)\right\} \prod_{1 \leq j<k \leq M}\left(1-\gamma_{j} \gamma_{k}\right) .
\end{aligned}
$$

Proof. Since the type II domain wall boundary partition functions $Z_{M}^{\mathrm{II}}\left(z_{1}, \ldots, z_{M} \mid \gamma_{1}, \ldots, \gamma_{M}\right)$ are special cases of the type II wavefunctions $\Phi_{M, M}^{\mathrm{II}}\left(z_{1}, \ldots, z_{M}\left|\gamma_{1}, \ldots, \gamma_{M}\right| 1, \ldots, M\right)$ (7.1), it is enough to prove the following special case of Proposition 6.3.

Proposition 7.2. The type II domain wall boundary partition functions $Z_{M}^{\mathrm{II}}\left(z_{1}, \ldots, z_{M} \mid \gamma_{1}, \ldots, \gamma_{M}\right)$ satisfies the following properties.

(1) The type II domain wall boundary partition functions $Z_{M}^{\mathrm{II}}\left(z_{1}, \ldots, z_{M} \mid \gamma_{1}, \ldots, \gamma_{M}\right)$ is a polynomial of degree $2 M-1$ in $\gamma_{M}$.

(2) The following form

$$
\frac{Z_{M}^{\mathrm{II}}\left(z_{1}, \ldots, z_{M} \mid \gamma_{1}, \ldots, \gamma_{M}\right)}{\prod_{j=1}^{M} z_{j}^{j-1 / 2-M}\left(1-\sqrt{-t} z_{j}\right) \prod_{1 \leq j<k \leq M}\left(1+t z_{j} z_{k}\right)\left(1+t z_{j} z_{k}^{-1}\right)},
$$

is symmetric with respect to $z_{1}, \ldots, z_{M}$, and is invariant under the exchange $z_{i} \longleftrightarrow z_{i}^{-1}$ for $i=1, \ldots, M$.

(3) The following recursive relations between the type II domain wall boundary partition functions hold:

$$
\begin{aligned}
& \left.Z_{M}^{\mathrm{II}}\left(z_{1}, \ldots, z_{M} \mid \gamma_{1}, \ldots, \gamma_{M}\right)\right|_{\gamma_{M}=z_{M}} \\
& =z_{M}^{-1 / 2} \prod_{j=1}^{M-1}\left(t z_{M} z_{j}+1\right) \prod_{j=1}^{M-1}\left(t+z_{M} z_{j}^{-1}\right) \prod_{j=1}^{M-1}\left(\left(1-\alpha_{j} \gamma_{j}\right) z_{M}^{-1}+\alpha_{j}\right) \prod_{j=1}^{M-1}\left(1-\gamma_{j} z_{M}\right) \\
& \quad \times Z_{M-1}^{\mathrm{II}}\left(z_{1}, \ldots, z_{M-1} \mid \gamma_{1}, \ldots, \gamma_{M-1}\right)
\end{aligned}
$$


(4) The following holds for the case $M=1$ :

$$
Z_{1}^{\mathrm{II}}\left(z \mid \gamma_{1}\right)=\frac{z^{1 / 2}(1-\sqrt{-t} z)}{z^{2}-1} \sum_{\tau= \pm 1} \tau\left(z^{\tau}+\sqrt{-t}\right)\left(1-\gamma_{1} z^{-\tau}\right) .
$$

It is easy to see that the right hand side of 7.2 satisfies all the properties listed in Proposition 7.2 .

We now derive the dual Cauchy formula for the generalized Whittaker functions.

Theorem 7.3. The following dual Cauchy formula holds for the generalized Whittaker functions with sets variables $\{x\}_{N}=\left\{x_{1}, \ldots, x_{N}\right\},\{y\}_{M}=\left\{y_{1}, \ldots, y_{M}\right\},\{\alpha\}=\left\{\alpha_{1}, \ldots, \alpha_{N+M}\right\}$, $\{\gamma\}=\left\{\gamma_{1}, \ldots, \gamma_{N+M}\right\}$,

$$
\begin{aligned}
& \sum_{\lambda \subseteq M^{N}} o_{\lambda}^{+}\left(\{x\}_{N}|\{\alpha\}|\{\gamma\} \mid t\right) o_{\hat{\lambda}}^{-}\left(\{y\}_{M}|\{-\alpha\}|\{-\gamma\} \mid t\right) \\
& =\prod_{j=1}^{M} y_{j}^{-N} \prod_{j=1}^{M+N}\left(1+\sqrt{-t} \gamma_{j}\right) \prod_{j=1}^{N} \prod_{k=1}^{M}\left(1+x_{j} y_{k}\right)\left(1+x_{j}^{-1} y_{k}\right) \\
& \quad \times \prod_{1 \leq j<k \leq N+M}\left\{1+\alpha_{j}\left(\gamma_{k}-\gamma_{j}\right)\right\} \prod_{1 \leq j<k \leq N+M}\left(1-\gamma_{j} \gamma_{k}\right),
\end{aligned}
$$

where $\{-\alpha\}=\left\{-\alpha_{1}, \ldots,-\alpha_{N+M}\right\},\{-\gamma\}=\left\{-\gamma_{1}, \ldots,-\gamma_{N+M}\right\}$ and $\hat{\lambda}=\left(\hat{\lambda}_{1}, \ldots, \hat{\lambda}_{M}\right)$ is the partition of the Young diagram $\lambda=\left(\lambda_{1}, \ldots, \lambda_{N}\right)$ given by

$$
\hat{\lambda}_{i}=\left|\left\{j \mid \lambda_{j} \leq M-i\right\}\right| \text {. }
$$

Proof. The type II domain wall boundary partition functions $Z_{M}^{\mathrm{II}}\left(z_{1}, \ldots, z_{M} \mid \gamma_{1}, \ldots, \gamma_{M}\right)$ have the factorized form 7.2$)$ in Theorem 7.1 on one hand.

On the other hand, one can evaluate the domain wall boundary partition functions by inserting the completeness relation and using the correspondences between the wavefunctions, the dual wavefunctions and the generalized Whittaker functions $(6.16)$ and $(6.17)$ as

$$
\begin{aligned}
Z_{M}^{\mathrm{II}}\left(z_{1}, \ldots, z_{M} \mid \gamma_{1}, \ldots, \gamma_{M}\right) & \\
= & \sum_{x \sqcup \bar{x}=\{1,2, \ldots, M\}} \bar{\Phi}_{M, M-N}^{\mathrm{I}}\left(z_{1}, \ldots, z_{M-N}\left|\gamma_{1}, \ldots, \gamma_{M}\right| \overline{x_{1}}, \ldots, \overline{x_{M-N}}\right) \\
& \times \Phi_{M, N}^{\mathrm{I}}\left(z_{M-N+1}, \ldots, z_{M}\left|\gamma_{1}, \ldots, \gamma_{M}\right| x_{1}, \ldots, x_{N}\right), \\
= & \sum_{\lambda \subseteq(M-N)^{N}} t^{N(M-N)} \prod_{j=1}^{M-N} z_{j}^{j-1 / 2-M+N}\left(1-\sqrt{-t} z_{j}\right) \prod_{1 \leq j<k \leq M-N}\left(1+t z_{j} z_{k}\right)\left(1+t z_{j} z_{k}^{-1}\right) \\
& \times o_{\bar{\lambda}}^{-}\left(t z_{1}, \ldots, t z_{M-N}|\{-\alpha\}|\{-\gamma\} \mid t\right) \\
& \times \prod_{j=M-N+1}^{M} z_{j}^{j-1 / 2-M}\left(1-\sqrt{-t} z_{j}\right) \prod_{M-N+1 \leq j<k \leq M}\left(1+t z_{j} z_{k}\right)\left(1+t z_{j} z_{k}^{-1}\right) \\
& \times o_{\lambda}^{+}\left(z_{M-N+1}, \ldots, z_{M}|\{\alpha\}|\{\gamma\} \mid t\right) .
\end{aligned}
$$


Comparing the two ways of evaluations $(7.8)$ and $(7.2)$ and cancelling common factors leads to

$$
\begin{aligned}
& \prod_{j=1}^{M-N}\left(t z_{j}\right)^{-N} \prod_{j=1}^{M}\left(1+\sqrt{-t} \gamma_{j}\right) \prod_{\substack{1 \leq j \leq M-N \\
M-N+1 \leq k \leq M}}\left(1+t z_{j} z_{k}\right)\left(1+t z_{j} z_{k}^{-1}\right) \\
& \quad \times \prod_{1 \leq j<k \leq M}\left(1+\alpha_{j}\left(\gamma_{k}-\gamma_{j}\right)\right) \prod_{\substack{1 \leq j<k \leq M\\
}}\left(1-\gamma_{j} \gamma_{k}\right) \\
& \quad=\sum_{\lambda \subseteq(M-N)^{N}} o_{\bar{\lambda}}^{-}\left(t z_{1}, \ldots, t z_{M-N}|\{-\alpha\}|\{-\gamma\} \mid t\right) o_{\lambda}^{+}\left(z_{M-N+1}, \ldots, z_{M}|\{\alpha\}|\{\gamma\} \mid t\right)
\end{aligned}
$$

which, after some reparametrization, can be rewritten in the form (7.6).

\section{Conclusion}

In this paper, we introduced and studied in detail generalizations of the free-fermionic sixvertex model under two types of reflecting boundary which were recently introduced by Ivanov and Brubaker-Bump-Chinta-Gunnells. We derived the explicit forms of the wavefunctions and the dual wavefunctions by using the Izergin-Korepin technique, a technique which belongs to a class of the quantum inverse scattering method. For the case which one uses the $K$ matrix by Ivanov [43, 44] at the boundary, we showed the wavefunctions can be expressed as a product of factors and the generalized symplectic Schur functions, which generalizes the correspondence by Ivanov. For the case which one uses the $K$-matrix by Brubaker-BumpChinta-Gunnells [46], we proved the wavefunctions are expressed as a product of factors and generalizations of the Whittaker functions introduced by Bump-Friedberg-Hoffstein [60]. The correspondence reduces to the conjecture by Brubaker-Bump-Chinta-Gunnells [46] when all the factorial parameters are set to zero.

We also derived the factorized form of the domain wall boundary partition functions for both models. As a consequence, we derived the dual Cauchy formulas for the generalized type $B$ Whittaker functions and the symplectic Schur functions.

It is interesting to apply the analysis given in this paper to other boundary conditions. Formulating analogues of the wavefunctions by looking at the graphical representations of the partition functions introduced and studied by Kuperberg [9] or Brubaker-Schultz [47], and applying the Izergin-Korepin analysis to find the explicit forms, and deriving algebraic identities is an interesting problem to be studied. It should make the connections between number theory and integrable lattice models more fruitful.

\section{Acknowledgments}

This work was partially supported by grant-in-Aid for and Scientific Research (C) No. 18K03205 and No. 16K05468. The authors thank the referee for helpful comments and suggestions to improve the paper. 


\section{A Some calculations for Property (2) in Proposition 4.4 and 6.3}

Here we present some calculations for the proof of Property (2) in Proposition 4.4 and 6.3 . First let us show the commutation relation of the $B$-operators

$$
\mathcal{B}\left(z_{i}\right) \mathcal{B}\left(z_{j}\right)=\frac{z_{j}+t z_{i}}{z_{i}+t z_{j}} \mathcal{B}\left(z_{j}\right) \mathcal{B}\left(z_{i}\right)
$$

where this relation holds for both type I and II $B$-operators. This commutation relation can be proven by use of the graphical representation in the following manner.


Let us explain in order. For the first equality, we have used the elements of the $R$-matrices shown in Figure 3. In going from the first to the second line, we have applied the Yang-Baxter 
relation 3.2 to commute the $B$-operators. Finally the following "caduceus relation"

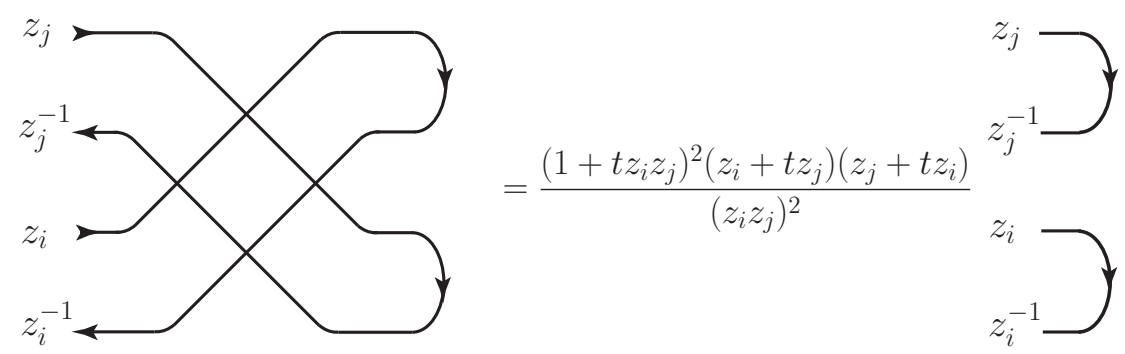

have been applied to the last line. The caduceus relation can be easily proved by the reflection equation (3.4):
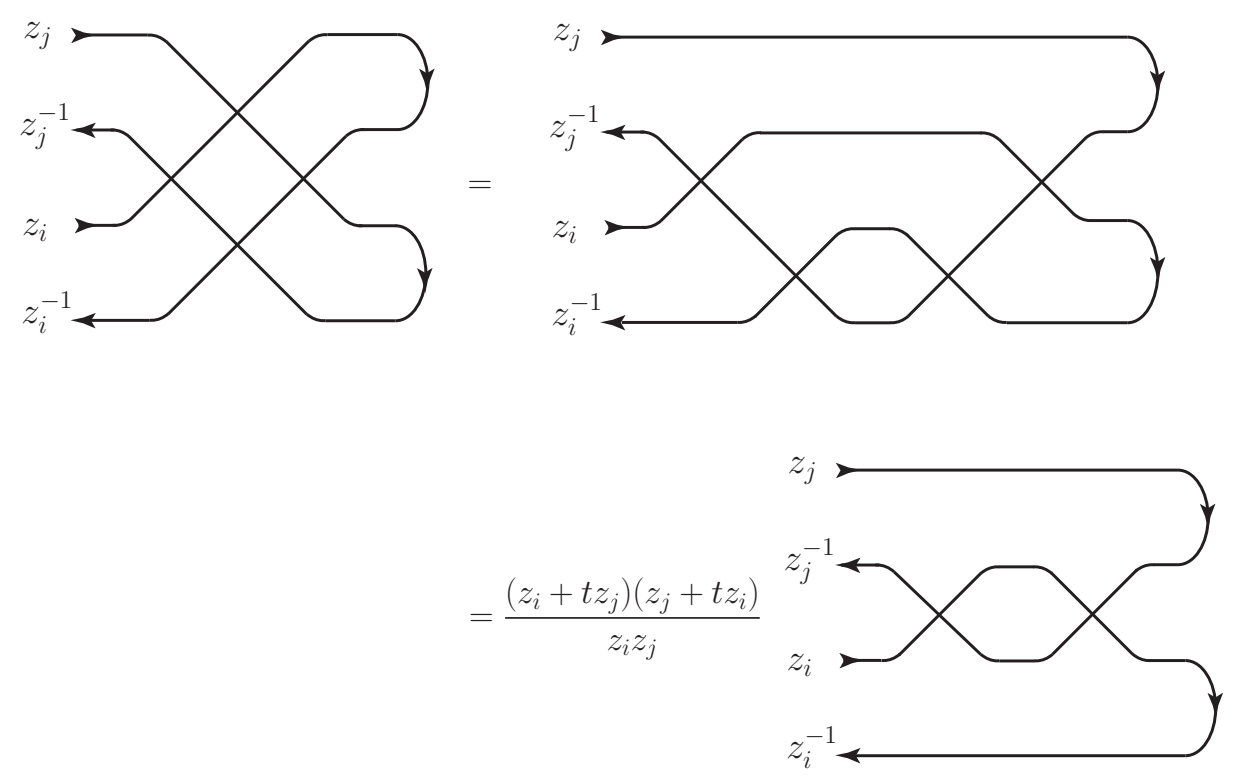

Here the reflection equation has been applied in the first equality. In going from the first to the second equality and the second to the third equality, we have applied the following unitarity relations.
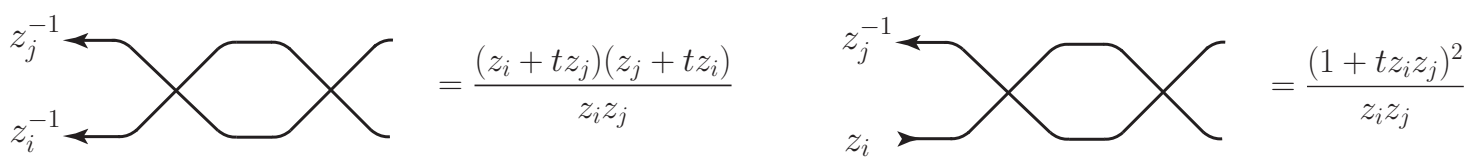

Thus the quantity

$$
\frac{\mathcal{B}\left(z_{N}\right) \cdots \mathcal{B}\left(z_{1}\right)}{\prod_{1 \leq j<k \leq N}\left(z_{k}+t z_{j}\right)}
$$

are symmetric with respect to the variables $z_{1}, \ldots, z_{N}$, and hence 4.6) and 6.5 are symmetric with respect to $z_{1}, \ldots, z_{N}$. 
Next along the procedure in [43, 44, we show the quantities (4.6) and (4.11) are invariant under the exchange $z_{i} \longleftrightarrow z_{i}^{-1}$. Due to the symmetry of A.6) with respect to $z_{1}, \ldots, z_{N}$, it is sufficient to consider the case $z_{N} \longleftrightarrow z_{N}^{-1}$ in Figure 7. In fact, the type $\Gamma L$-operator on the last row in Figure 7 can be replaced by the second type (type $\Delta$ ) $L$-operator $\widetilde{L}$ as follows. Because the elements of the $L$ appearing on the last row are only the following three types:

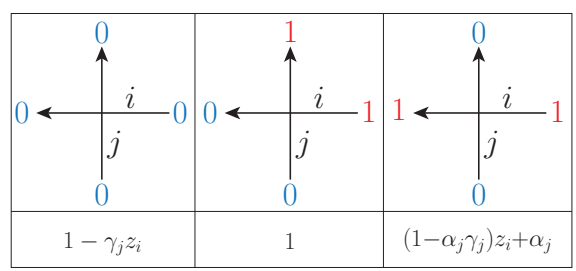

which are equivalent to the elements of $\widetilde{L}$ :

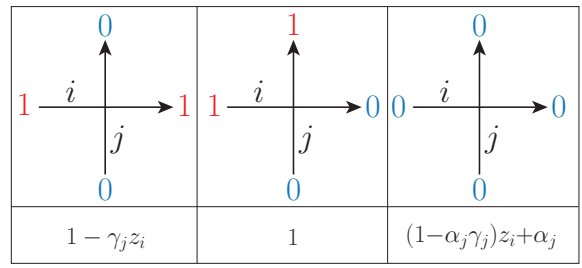

Thus we replace $\widetilde{L}$ to $L$ with $0 \leftrightarrow 1$ without changing the wavefunctions.

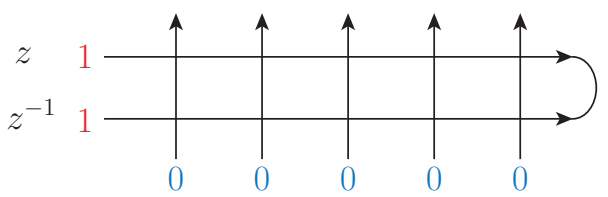

where the type I and type II $K$-matrices are, respectively, modified as

Multiplying the type $\Delta \Delta R$-matrix and using the Yang-Baxter relation (3.2), we obtain
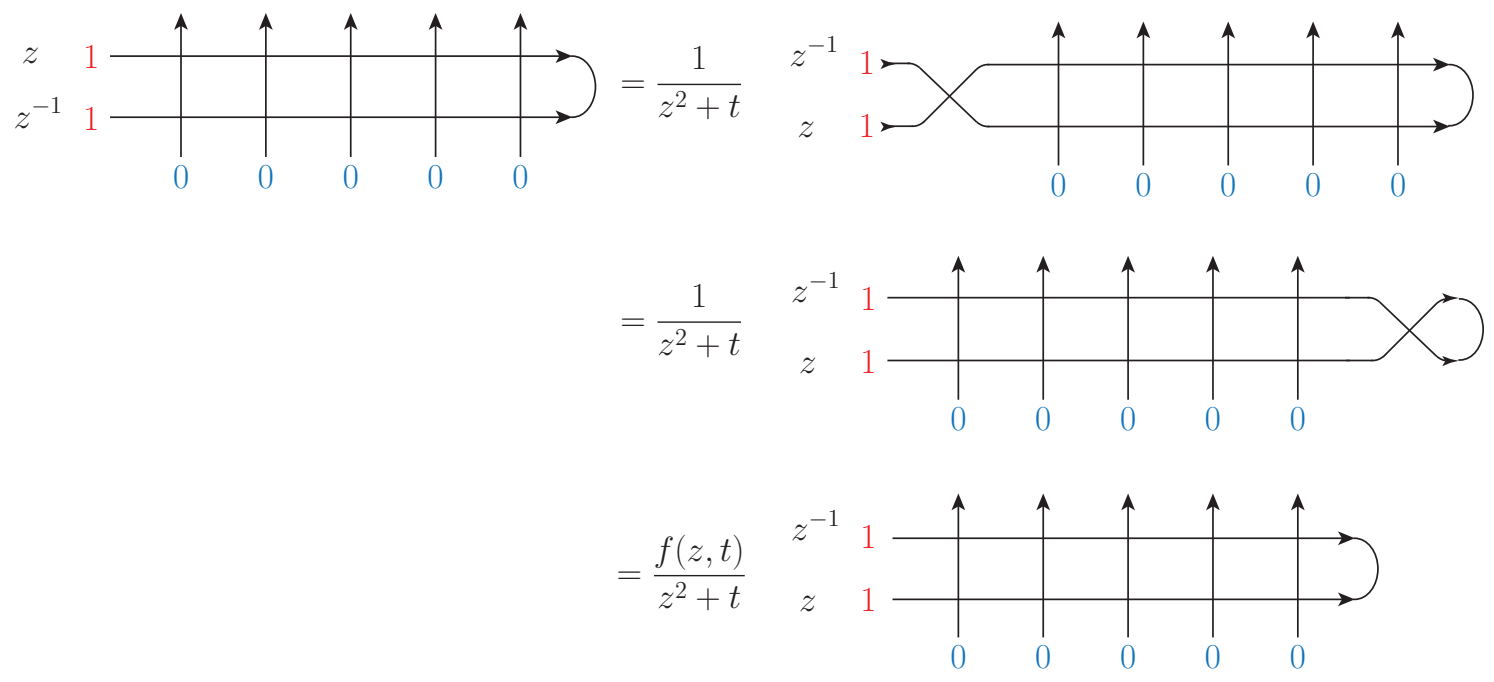

(A.8)

where we have used the elements of the $R$-matrix (see Figure 3 ) and the following relation called "fish relation" [43, 44]:

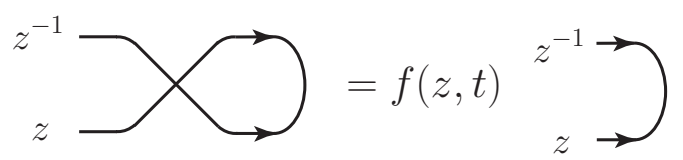

The overall factor $f(z, t)$ is given by

$$
f(z, t)=\left\{\begin{array}{ll}
1+t z^{2} & \text { for type I } \\
(\sqrt{-t}+z)(1-\sqrt{-t} z) & \text { for type II }
\end{array} .\right.
$$

Namely, the following expression holds:

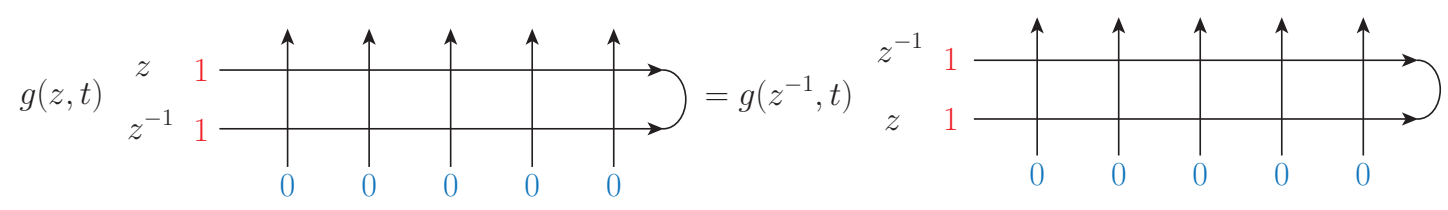

with

$$
g(z, t)=\left\{\begin{array}{ll}
\frac{z}{1+t z^{2}} & \text { for type I } \\
\frac{z^{1 / 2}}{1-\sqrt{-t} z} & \text { for type II }
\end{array} .\right.
$$

Therefore, the quantities (4.6) and (6.5) are invariant under $z_{i} \longleftrightarrow z_{i}^{-1}$. 


\section{B Some calculations for the dual wavefunctions}

Let us show some computations about the proof of the dual wavefunctions. To prove $(4.19)$ in

Theorem 4.7, one must show that the following functions $\bar{F}_{M, N}^{\mathrm{I}}\left(z_{N}, \ldots, z_{1}\left|\gamma_{1}, \ldots, \gamma_{M}\right| \overline{x_{1}}, \ldots, \overline{x_{N}}\right)$ satisfy 4.12 in Proposition 4.5 .

$$
\begin{aligned}
& \bar{F}_{M, N}^{\mathrm{I}}\left(z_{N}, \ldots, z_{1}\left|\gamma_{1}, \ldots, \gamma_{M}\right| \overline{x_{1}}, \ldots, \overline{x_{N}}\right) \\
&:= t^{N(M-N)} \prod_{j=1}^{N} z_{j}^{j-1-N}\left(1+t z_{j}^{2}\right) \prod_{1 \leq j<k \leq N}\left(1+t z_{j} z_{k}\right)\left(1+t z_{j} z_{k}^{-1}\right) \\
& \times\left. s p_{\bar{\lambda}}\left(\{t z\}_{N}|\{-\bar{\alpha}\}|\{-\bar{\gamma}\}\right)\right|_{z_{i} \rightarrow z_{N+1-i}} \\
&= \frac{t^{N(M-N)} \prod_{j=1}^{N} z_{N+1-j}^{j-1-N}\left(1+t z_{N+1-j}^{2}\right) \prod_{1 \leq k<j \leq N}\left(1+t z_{j} z_{k}\right)\left(1+t z_{j} z_{k}^{-1}\right)}{(-1)^{N} \prod_{j=1}^{N}\left(t z_{j}\right)^{j-1-N}\left(1-t^{2} z_{j}^{2}\right) \prod_{1 \leq j<k \leq N}\left(1-t^{2} z_{j} z_{k}\right)\left(-1+z_{j} z_{k}^{-1}\right)} \\
& \times \sum_{\sigma \in S_{N}} \sum_{\tau_{1}= \pm 1, \ldots, \tau_{N}= \pm 1}(-1)^{\sigma}(-1)^{|\tau|} \prod_{j=1}^{N} \prod_{k=0}^{\overline{x_{j}}-1}\left\{-\alpha_{k}+\left(1-\alpha_{k} \gamma_{k}\right)\left(t z_{\sigma(j)}\right)^{\tau_{\sigma(j)}}\right\} \\
& \times \prod_{j=1}^{N} \prod_{k=\overline{x_{j}}+1}^{M}\left\{1+\gamma_{k}\left(t z_{\sigma(j)}\right)^{\tau_{\sigma(j)}}\right\} \prod_{j=1}^{N} \prod_{k=1}^{M}\left\{1+\gamma_{k}\left(t z_{\sigma(j)}\right)^{-\tau_{\sigma(j)}}\right\} .
\end{aligned}
$$

To check this, one first rewrites (B.1) as

$$
\begin{aligned}
\bar{F}_{M, N}^{\mathrm{I}}\left(z_{N}, \ldots, z_{1}\left|\gamma_{1}, \ldots, \gamma_{M}\right| \overline{x_{1}}, \ldots, \overline{x_{N}}\right) \\
=\frac{t^{N(M-N)} \prod_{j=1}^{N}\left(1+t z_{j}^{2}\right) \prod_{1 \leq j<k \leq N}\left(1+t z_{j} z_{k}\right)\left(1+t z_{j}^{-1} z_{k}\right)}{t^{-N(N+1) / 2}(-1)^{N} \prod_{j=1}^{N}\left(1-t^{2} z_{j}^{2}\right) \prod_{1 \leq j<k \leq N}\left(1-t^{2} z_{j} z_{k}\right)\left(1-z_{j}^{-1} z_{k}\right)} \\
\quad \times \sum_{\sigma \in S_{N}} \sum_{\tau_{1}= \pm 1, \ldots, \tau_{N}= \pm 1}(-1)^{\sigma}(-1)^{|\tau|} \prod_{j=1}^{N} \prod_{k=0}^{\overline{x_{j}}-1}\left(-\alpha_{k}+\left(1-\alpha_{k} \gamma_{k}\right)\left(t z_{\sigma(j)}\right)^{\tau_{\sigma(j)}}\right) \\
\quad \times \prod_{j=1}^{N} \prod_{k=\overline{x_{j}}+1}^{M}\left\{1+\gamma_{k}\left(t z_{\sigma(j)}\right)^{\tau_{\sigma(j)}}\right\} \prod_{j=1}^{N} \prod_{k=1}^{M}\left\{1+\gamma_{k}\left(t z_{\sigma(j)}\right)^{-\tau_{\sigma(j)}}\right\}
\end{aligned}
$$

Specializing $\gamma_{M}=-t^{-1} z_{N}^{-1}$ and $\overline{x_{N}}=M$, only the summands satisfying $\sigma(N)=N, \tau_{N}=$ +1 in $B .2$ survive, from which we find that $\left.\bar{F}_{M, N}^{\mathrm{I}}\left(z_{1}, \ldots, z_{N}\left|\gamma_{1}, \ldots, \gamma_{M}\right| \overline{x_{1}}, \ldots, \overline{x_{N}}\right)\right|_{\gamma_{M}=-t^{-1} z_{N}^{-1}}$ 
can be rewritten as

$$
\begin{aligned}
\left.\bar{F}_{M, N}^{\mathrm{I}}\left(z_{1}, \ldots, z_{N}\left|\gamma_{1}, \ldots, \gamma_{M}\right| \overline{x_{1}}, \ldots, \overline{x_{N}}\right)\right|_{\gamma_{M}=-t^{-1} z_{N}^{-1}} \\
=-t^{M} \frac{1+t z_{N}^{2}}{1-t^{2} z_{N}^{2}} \frac{\prod_{j=1}^{N-1}\left(1+t z_{j} z_{N}\right)\left(1+t z_{j}^{-1} z_{N}\right)}{\prod_{j=1}^{N-1}\left(1-t^{2} z_{j} z_{N}\right)\left(1-z_{j}^{-1} z_{N}\right)} \\
\quad \times \frac{t^{(N-1)(M-1-(N-1))} \prod_{j=1}^{N-1}\left(1+t z_{j}^{2}\right) \prod_{1 \leq j<k \leq N-1}\left(1+t z_{j} z_{k}\right)\left(1+t z_{j}^{-1} z_{k}\right)}{t^{-(N-1) N / 2}(-1)^{N-1} \prod_{j=1}^{N-1}\left(1-t^{2} z_{j}^{2}\right) \prod_{1 \leq j<k \leq N-1}\left(1-t^{2} z_{j} z_{k}\right)\left(1-z_{j}^{-1} z_{k}\right)}(-1)^{\sigma}(-1)^{|\tau|} \\
\quad \times \sum_{\sigma \in S_{N-1} \tau_{1}= \pm 1, \ldots, \tau_{N-1}= \pm 1} \sum_{j=1}^{N-1} \overline{x_{j}-1}\left\{\prod_{k=0}^{M-\alpha}\left\{-\alpha_{k}+\left(1-\alpha_{k} \gamma_{k}\right)\left(t z_{\sigma(j)}\right)^{\tau_{\sigma(j)}}\right\} \prod_{k=0}^{M-1}\left\{-\alpha_{k}+t\left(1-\alpha_{k} \gamma_{k}\right) z_{N}\right\}\right. \\
\quad \times \prod_{j=1}^{N-1} \prod_{k=\overline{x_{j}}+1}^{M-1}\left\{1+\gamma_{k}\left(t z_{\sigma(j)}\right)^{\tau_{\sigma(j)}}\right\} \prod_{j=1}^{N-1}\left\{1-\frac{\left(t z_{\sigma(j)}\right)^{\tau_{\sigma(j)}}}{t z_{N}}\right\} \\
\quad \times\left(1-\frac{1}{t^{2} z_{N}^{2}}\right) \prod_{k=1}^{M-1}\left(1+\frac{\gamma_{k}}{t z_{N}}\right) \prod_{j=1}^{N-1}\left\{1-\frac{\left(t z_{\sigma(j)}\right)^{-\tau_{\sigma(j)}}}{t z_{N}}\right\} \prod_{j=1}^{N-1} \prod_{k=1}^{M-1}\left\{1+\gamma_{k}\left(t z_{\sigma(j)}\right)^{-\tau_{\sigma(j)}}\right\} .
\end{aligned}
$$

Using the identity

$$
\prod_{j=1}^{N-1}\left\{1-\frac{\left(t z_{\sigma(j)}\right)^{\tau_{\sigma(j)}}}{t z_{N}}\right\}\left\{1-\frac{\left(t z_{\sigma(j)}\right)^{-\tau_{\sigma(j)}}}{t z_{N}}\right\}=\prod_{j=1}^{N-1}\left(1-\frac{z_{j}}{z_{N}}\right)\left(1-\frac{1}{t^{2} z_{N} z_{j}}\right),
$$

to simplify B.3 leads to

$$
\begin{aligned}
&\left.\bar{F}_{M, N}^{\mathrm{I}}\left(z_{1}, \ldots, z_{N}\left|\gamma_{1}, \ldots, \gamma_{M}\right| \overline{x_{1}}, \ldots, \overline{x_{N}}\right)\right|_{\gamma_{M}=-t^{-1} z_{N}^{-1}} \\
&=\prod_{j=1}^{N}\left(1+\frac{1}{t z_{N} z_{j}}\right) \prod_{j=1}^{N-1}\left(1+\frac{z_{j}}{t z_{N}}\right) \prod_{j=0}^{M-1}\left\{t\left(1-\alpha_{j} \gamma_{j}\right) z_{N}-\alpha_{j}\right\} \prod_{j=1}^{M-1}\left(t+\gamma_{j} z_{N}^{-1}\right) \\
& \times \frac{t^{(N-1)(M-1-(N-1))} \prod_{j=1}^{N-1}\left(1+t z_{j}^{2}\right) \prod_{1 \leq j<k \leq N-1}\left(1+t z_{j} z_{k}\right)\left(1+t z_{j}^{-1} z_{k}\right)}{t^{-(N-1) N / 2}(-1)^{N-1} \prod_{j=1}^{N-1}\left(1-t^{2} z_{j}^{2}\right) \prod_{1 \leq j<k \leq N-1}\left(1-t^{2} z_{j} z_{k}\right)\left(1-z_{j}^{-1} z_{k}\right)} \\
& \times \sum_{\sigma \in S_{N-1}} \sum_{\tau_{1}= \pm 1, \ldots, \tau_{N-1}= \pm 1}(-1)^{\sigma}(-1)^{|\tau|} \prod_{j=1}^{N-1} \prod_{k=0}^{\overline{x_{j}}-1}\left\{-\alpha_{k}+\left(1-\alpha_{k} \gamma_{k}\right)\left(t z_{\sigma(j)}\right)^{\tau_{\sigma(j)}}\right\} \\
& \times \prod_{j=1}^{N-1} \prod_{k=\overline{x_{j}}+1}^{M-1}\left\{1+\gamma_{k}\left(t z_{\sigma(j)}\right)^{\tau_{\sigma(j)}}\right\} \prod_{j=1}^{N-1} \prod_{k=1}^{M-1}\left\{1+\gamma_{k}\left(t z_{\sigma(j)}\right)^{-\tau_{\sigma(j)}}\right\} \\
&= \prod_{j=1}^{N}\left(1+\frac{1}{t z_{N} z_{j}}\right) \prod_{j=1}^{N-1}\left(1+\frac{z_{j}}{t z_{N}}\right) \prod_{j=0}^{M-1}\left\{t\left(1-\alpha_{j} \gamma_{j}\right) z_{N}-\alpha_{j}\right\} \prod_{j=1}^{M-1}\left(t+\gamma_{j} z_{N}^{-1}\right) \\
& \times \bar{F}_{M-1, N-1}^{\mathrm{I}}\left(z_{N-1}, \ldots, z_{1}\left|\gamma_{1}, \ldots, \gamma_{M-1}\right| \overline{x_{1}}, \ldots, \overline{x_{N-1}}\right),
\end{aligned}
$$


and we have shown $\bar{F}_{M, N}^{\mathrm{I}}\left(z_{N}, \ldots, z_{1}\left|\gamma_{1}, \ldots, \gamma_{M}\right| \overline{x_{1}}, \ldots, \overline{x_{N}}\right)$ satisfy 4.12 in Proposition 4.5 .

Similarly, to prove (6.17) in Theorem 6.6, one has to check that the following functions $\bar{F}_{M, N}^{\mathrm{II}}\left(z_{N}, \ldots, z_{1}\left|\gamma_{1}, \ldots, \gamma_{M}\right| \overline{x_{1}}, \ldots, \overline{x_{N}}\right)$ satisfy 6.10 in Proposition 6.4

$$
\begin{aligned}
& \bar{F}_{M, N}^{\mathrm{II}}\left(z_{N}, \ldots, z_{1}\left|\gamma_{1}, \ldots, \gamma_{M}\right| \overline{x_{1}}, \ldots, \overline{x_{N}}\right) \\
&:= t^{N(M-N)} \prod_{j=1}^{N} z_{j}^{j-1 / 2-N}\left(1-\sqrt{-t} z_{j}\right) \prod_{1 \leq j<k \leq N}\left(1+t z_{j} z_{k}\right)\left(1+t z_{j} z_{k}^{-1}\right) \\
& \times\left. o_{\bar{\lambda}}^{-}\left(\{t z\}_{N}|\{-\alpha\}|\{-\gamma\} \mid t\right)\right|_{z_{i} \rightarrow z_{N+1-i}} \\
&= \frac{t^{N(M-N)} \prod_{j=1}^{N} z_{N+1-j}^{j-1 / 2-N}\left(1-\sqrt{-t} z_{N+1-j}\right) \prod_{1 \leq k<j \leq N}\left(1+t z_{j} z_{k}\right)\left(1+t z_{j} z_{k}^{-1}\right)}{(-1)^{N} \prod_{j=1}^{N}\left(t z_{j}\right)^{j-1-N}\left(1-t^{2} z_{j}^{2}\right) \prod_{1 \leq j<k \leq N}\left(1-t^{2} z_{j} z_{k}\right)\left(-1+z_{j} z_{k}^{-1}\right)} \\
& \times \sum_{\sigma \in S_{N}} \sum_{j= \pm 1, \ldots, \tau_{N}= \pm 1}(-1)^{\sigma}(-1)^{|\tau|} \prod_{j=1}^{N}\left\{\left(t z_{\sigma(j)}\right)^{\tau_{\sigma(j)}}-\sqrt{-t}\right\} \\
& \times \prod_{j=1}^{N} \prod_{k=1}^{\overline{x_{j}}-1}\left\{-\alpha_{k}+\left(1-\alpha_{k} \gamma_{k}\right)\left(t z_{\sigma(j)}\right)^{\left.\tau_{\sigma(j)}\right\}}\right. \\
& \times \prod_{j=1}^{N} \prod_{k=\overline{x_{j}}+1}^{M}\left\{1+\gamma_{k}\left(t z_{\sigma(j)}\right)^{\tau_{\sigma(j)}}\right\} \prod_{j=1}^{N} \prod_{k=1}^{M}\left\{1+\gamma_{k}\left(t z_{\sigma(j)}\right)^{-\tau_{\sigma(j)}}\right\} .
\end{aligned}
$$

We again rewrite $(\overline{B .6})$ as

$$
\begin{aligned}
\bar{F}_{M, N}^{\mathrm{II}}\left(z_{N}, \ldots, z_{1}\left|\gamma_{1}, \ldots, \gamma_{M}\right| \overline{x_{1}}, \ldots, \overline{x_{N}}\right) \\
=\frac{t^{N(M-N)} \prod_{j=1}^{N} z_{j}^{1 / 2}\left(1-\sqrt{-t} z_{j}\right) \prod_{1 \leq j<k \leq N}\left(1+t z_{j} z_{k}\right)\left(1+t z_{j}^{-1} z_{k}\right)}{t^{-N(N+1) / 2}(-1)^{N} \prod_{j=1}^{N}\left(1-t^{2} z_{j}^{2}\right) \prod_{1 \leq j<k \leq N}\left(1-t^{2} z_{j} z_{k}\right)\left(1-z_{j}^{-1} z_{k}\right)} \\
\quad \times \sum_{\sigma \in S_{N}} \sum_{\tau_{1}= \pm 1, \ldots, \tau_{N}= \pm 1}(-1)^{\sigma}(-1)^{|\tau|} \prod_{j=1}^{N}\left\{\left(t z_{\sigma(j)}\right)^{\tau_{\sigma(j)}}-\sqrt{-t}\right\} \\
\quad \times \prod_{j=1}^{N} \prod_{k=1}^{\overline{x_{j}}-1}\left\{-\alpha_{k}+\left(1-\alpha_{k} \gamma_{k}\right)\left(t z_{\sigma(j)}\right)^{\tau_{\sigma(j)}}\right\} \\
\quad \times \prod_{j=1}^{N} \prod_{k=\overline{x_{j}}+1}^{M}\left\{1+\gamma_{k}\left(t z_{\sigma(j)}\right)^{\tau_{\sigma(j)}}\right\} \prod_{j=1}^{N} \prod_{k=1}^{M}\left\{1+\gamma_{k}\left(t z_{\sigma(j)}\right)^{-\tau_{\sigma(j)}}\right\} .
\end{aligned}
$$

Only the summands satisfying $\sigma(N)=N, \tau_{N}=+1$ in B.7) survive after the substitution $\gamma_{M}=-t^{-1} z_{N}^{-1}$ and $\overline{x_{N}}=M$ from which we finds 


$$
\begin{aligned}
\left.\bar{F}_{M, N}^{\mathrm{II}}\left(z_{1}, \ldots, z_{N}\left|\gamma_{1}, \ldots, \gamma_{M}\right| \overline{x_{1}}, \ldots, \overline{x_{N}}\right)\right|_{\gamma_{M}=-t^{1} z_{N}^{1}} \\
=-t^{M} \frac{z_{N}^{1 / 2}\left(1-\sqrt{-t} z_{N}\right)}{1-t^{2} z_{N}^{2}} \frac{\prod_{j=1}^{N-1}\left(1+t z_{j} z_{N}\right)\left(1+t z_{j}^{-1} z_{N}\right)}{\prod_{j=1}^{N-1}\left(1-t^{2} z_{j} z_{N}\right)\left(1-z_{j}^{-1} z_{N}\right)} \\
\quad \times \frac{t^{(N-1)(M-1-(N-1))} \prod_{j=1}^{N-1} z_{j}^{1 / 2}\left(1-\sqrt{-t} z_{j}\right) \prod_{1 \leq j<k \leq N-1}\left(1+t z_{j} z_{k}\right)\left(1+t z_{j}^{-1} z_{k}\right)}{t^{-(N-1) N / 2}(-1)^{N-1} \prod_{j=1}^{N-1}\left(1-t^{2} z_{j}^{2}\right) \prod_{1 \leq j<k \leq N-1}\left(1-t^{2} z_{j} z_{k}\right)\left(1-z_{j}^{-1} z_{k}\right)} \\
\quad \times \sum_{\sigma \in S_{N-1} \tau_{1}= \pm 1, \ldots, \tau_{N-1}= \pm 1}(-1)^{\sigma}(-1)^{|\tau|}\left(t z_{N}-\sqrt{-t}\right) \prod_{j=1}^{N-1}\left\{\left(t z_{\sigma(j)}\right)^{\tau_{\sigma(j)}}-\sqrt{-t}\right\} \\
\quad \times \prod_{j=1}^{N-1} \prod_{k=1}^{\overline{x_{j}}-1}\left\{-\alpha_{k}+\left(1-\alpha_{k} \gamma_{k}\right)\left(t z_{\sigma(j)}\right)^{\tau_{\sigma(j)}}\right\} \prod_{k=1}^{M-1}\left\{-\alpha_{k}+t\left(1-\alpha_{k} \gamma_{k}\right) z_{N}\right\} \\
\quad \times \prod_{j=1}^{N-1} \prod_{k=\overline{x_{j}}+1}^{M-1}\left\{1+\gamma_{k}\left(t z_{\sigma(j)}\right)^{\tau_{\sigma(j)}}\right\} \prod_{j=1}^{N-1}\left\{1-\frac{\left(t z_{\sigma(j)}\right)^{\tau} \sigma(j)}{t z_{N}}\right\} \\
\quad \times\left(1-\frac{1}{t^{2} z_{N}^{2}}\right) \prod_{k=1}^{M-1}\left(1+\frac{\gamma_{k}}{t z_{N}}\right) \prod_{j=1}^{N-1}\left\{1-\frac{\left(t z_{\sigma(j)}\right)^{-\tau_{\sigma(j)}}}{t z_{N}}\right\} \prod_{j=1}^{N-1} \prod_{k=1}^{M-1}\left\{1+\gamma_{k}\left(t z_{\sigma(j)}\right)^{-\tau_{\sigma(j)}}\right\} .
\end{aligned}
$$

We again use the identity

$$
\prod_{j=1}^{N-1}\left\{1-\frac{\left(t z_{\sigma(j)}\right)^{\tau_{\sigma(j)}}}{t z_{N}}\right\}\left\{1-\frac{\left(t z_{\sigma(j)}\right)^{-\tau_{\sigma(j)}}}{t z_{N}}\right\}=\prod_{j=1}^{N-1}\left(1-\frac{z_{j}}{z_{N}}\right)\left(1-\frac{1}{t^{2} z_{N} z_{j}}\right),
$$

to simplify B.8 as

$$
\begin{aligned}
& \left.\bar{F}_{M, N}^{\mathrm{II}}\left(z_{1}, \ldots, z_{N}\left|\gamma_{1}, \ldots, \gamma_{M}\right| \overline{x_{1}}, \ldots, \overline{x_{N}}\right)\right|_{\gamma_{M}=-t^{-1} z_{N}^{-1}} \\
& =-\sqrt{-t} z_{N}^{1 / 2} \prod_{j=1}^{N}\left(1+\frac{1}{t z_{N} z_{j}}\right) \prod_{j=1}^{N-1}\left(1+\frac{z_{j}}{t z_{N}}\right) \prod_{j=1}^{M-1}\left\{t\left(1-\alpha_{j} \gamma_{j}\right) z_{N}-\alpha_{j}\right\} \prod_{j=1}^{M-1}\left(t+\gamma_{j} z_{N}^{-1}\right) \\
& \quad \times \frac{t^{(N-1)(M-1-(N-1))} \prod_{j=1}^{N-1} z_{j}^{1 / 2}\left(1-\sqrt{-t} z_{j}\right) \prod_{1 \leq j<k \leq N-1}\left(1+t z_{j} z_{k}\right)\left(1+t z_{j}^{-1} z_{k}\right)}{t^{-(N-1) N / 2}(-1)^{N-1} \prod_{j=1}^{N-1}\left(1-t^{2} z_{j}^{2}\right) \prod_{1 \leq j<k \leq N-1}\left(1-t^{2} z_{j} z_{k}\right)\left(1-z_{j}^{-1} z_{k}\right)} \\
& \quad \times \sum_{\sigma \in S_{N-1}} \sum_{\tau_{1}= \pm 1, \ldots, \tau_{N-1}= \pm 1}(-1)^{\sigma}(-1)^{|\tau|} \prod_{j=1}^{N-1}\left\{\left(t z_{\sigma(j)}\right)^{\tau_{\sigma(j)}}-\sqrt{-t}\right\} \\
& \quad \times \prod_{j=1}^{N-1} \prod_{k=0}^{\overline{x_{j}}-1}\left\{-\alpha_{k}+\left(1-\alpha_{k} \gamma_{k}\right)\left(t z_{\sigma(j)}\right)^{\tau_{\sigma(j)}}\right\} \\
& \quad \times \prod_{j=1}^{N-1} \prod_{k=\overline{x_{j}}+1}^{M-1}\left\{1+\gamma_{k}\left(t z_{\sigma(j)}\right)^{\tau_{\sigma(j)}}\right\} \prod_{j=1}^{N-1} \prod_{k=1}^{M-1}\left\{1+\gamma_{k}\left(t z_{\sigma(j)}\right)^{-\tau_{\sigma(j)}}\right\}
\end{aligned}
$$




$$
\begin{aligned}
= & -\sqrt{-t} z_{N}^{1 / 2} \prod_{j=1}^{N}\left(1+\frac{1}{t z_{N} z_{j}}\right) \prod_{j=1}^{N-1}\left(1+\frac{z_{j}}{t z_{N}}\right) \prod_{j=1}^{M-1}\left\{t\left(1-\alpha_{j} \gamma_{j}\right) z_{N}-\alpha_{j}\right\} \prod_{j=1}^{M-1}\left(t+\gamma_{j} z_{N}^{-1}\right) \\
& \times \bar{F}_{M-1, N-1}^{\mathrm{II}}\left(z_{N-1}, \ldots, z_{1}\left|\gamma_{1}, \ldots, \gamma_{M-1}\right| \overline{x_{1}}, \ldots, \overline{x_{N-1}}\right),
\end{aligned}
$$

and we have shown $\bar{F}_{M, N}^{\mathrm{II}}\left(z_{N}, \ldots, z_{1}\left|\gamma_{1}, \ldots, \gamma_{M}\right| \overline{x_{1}}, \ldots, \overline{x_{N}}\right)$ satisfy 6.10 in Proposition 6.4

\section{References}

[1] V. Drinfeld, Hopf algebras and the quantum Yang-Baxter equation, Sov. math. Dokl. 32 (1985) 254.

[2] M, Jimbo, A $q$-difference analogue of $U(g)$ and the Yang-Baxter equation, Lett. Math. Phys. 10 (1985) 63.

[3] L.D. Faddeev, E.K. Sklyanin, L.A. Takhtajan, The Quantum Inverse Problem Method. 1, Theor. Math. Phys. 40 (1979) 194.

[4] R.J. Baxter, Exactly Solved Models in Statistical Mechanics, Academic Press, London, 1982.

[5] V.E. Korepin, N.M. Bogoliubov, A.G. Izergin, Quantum Inverse Scattering Method and Correlation functions, Cambridge University Press, Cambridge, 1993.

[6] V.E. Korepin, Calculation of Norms of Bethe Wavefunctions, Comm. Math. Phys. 86 (1982) 391.

[7] A. Izergin, Partition function of the 6-vertex model in a finite volume, Sov. phys. Dokl. $32(1987) 878$.

[8] D. Bressoud, Proofs and confirmations: The story of the alternating sign matrix conjecture, MAA Spectrum, Mathematical Association of America, Washington, DC, 1999.

[9] G. Kuperberg, Another proof of the alternating sign matrix conjecture, Int. Math. Res. Not. 3 (1996) 139.

[10] N. M. Bogoliubov, Boxed plane partitions as an exactly solvable boson model, J. Phys. A: Math. and Gen. 38 (2005) 9415.

[11] B. Brubaker, D. Bump, S. Friedberg, Schur polynomials and the Yang-Baxter equation, Comm. Math. Phys. 308 (2011) 281.

[12] D. Bump, P. McNamara, M. Nakasuji, Factorial Schur functions and the Yang-Baxter equation, Comm. Math. Univ. Sancti Pauli. 63 (2014) 23.

[13] A. Lascoux, The 6 Vertex Model and Schubert Polynomials, SIGMA 3 (2007) 029.

[14] P.J. McNamara, Factorial Schur functions via the six-vertex model, arXiv:0910.5288.

[15] C. Korff, C. Stroppel, The $s l(n)-W Z N W$ Fusion Ring: a combinatorial construction and a realisation as quotient of quantum cohomology, Adv. Math. 225 (2010) 200. 
[16] C. Korff Quantum cohomology via vicious and osculating walkers, Lett. Math. Phys. 104 (2014) 771.

[17] V. Gorbounov, Korff, Quantum Integrability and Generalised Quantum Schubert Calculus, Adv. Math. 313 (2017) 282.

[18] K. Motegi, K. Sakai, Vertex model, TASEP and Grothendieck polynomials, J. Phys. A: Math. and Theor. 46 (2013) 355201.

[19] K. Motegi, K. Sakai., K-theoretic boson-fermion correspondence and melting crystals, J. Phys. A: Math. and Theor. 47 (2014) 445202.

[20] K. Motegi, K. Sakai, S. Watanabe. Partition functions of integrable lattice models and combinatorics of symmetric polynomials, arXiv:1512.07955.

[21] D. Betea, M. Wheeler, Refined Cauchy and Littlewood Identities, Plane Partitions and Symmetry Classes of Alternating Sign Matrices, J. Comb. Theory, Series A. 137 (2016) 126.

[22] D. Betea, M. Wheeler, P. Zinn-Justin, Refined Cauchy/Littlewood identities and sixvertex model partition functions: II. Proofs and new conjectures, J. Alg. Comb. 42 (2015) 555 .

[23] M. Wheeler, P. Zinn-Justin, Refined Cauchy/Littlewood identities and six-vertex model partition functions: III. Deformed bosons, Adv. Math. 299 (2016) 543.

[24] A. Borodin, On a family of symmetric rational functions, Adv. Math. 306 (2017) 973.

[25] A. Borodin, L. Petrov, Higher spin six vertex model and symmetric rational functions, Sel. Math. New Series. 24 (2018) 1.

[26] Y. Takeyama, A deformation of affine Hecke algebra and integrable stochastic particle system, J. Phys. A: Math. and Theor. 47 (2014) 465203.

[27] Y. Takeyama, On the eigenfunctions for the multi-species $q$-Boson system, Funkcialaj Ekvacioj 61 (2018) 349.

[28] J.F. van Diejen, E. Emsiz, Orthogonality of Bethe Ansatz eigenfunctions for the Laplacian on a hyperoctahedral Weyl alcove, Comm. Math. Phys. 350 (2017) 1017.

[29] K. Motegi, Symmetric functions and wavefunctions of XXZ-type six-vertex models and elliptic Felderhof models by Izergin-Korepin analysis, J. Math. Phys. 59 (2018) 053505.

[30] T. Tokuyama, A generating function of strict Gelfand patterns and some formulas on characters of general linear groups, J. Math. Soc. Japan. 40 (1988) 671.

[31] S. Okada, Alternating sign matrices and some deformations of Weyl's denominator formula, J. Alg. Comb. 2 (1993) 155.

[32] A.M. Hamel, R.C. King, Tokuyamas identity for factorial Schur $P$ and $Q$ functions, Electronic J. Comb. 22 (2015) Paper P2.42. 
[33] B. Felderhof, Direct diagonalization of the transfer matrix of the zero-field free-fermion model, Physica 65 (1973) 421.

[34] J. Murakami, The free-fermion model in presence of field related to the quantum group $U_{q}\left(s l_{2}\right)$ of affine type and the multi-variable Alexander polynomial of links, Infinite analysis, Adv. Ser. in Math. Phys. 16B (1991) 765.

[35] T. Deguchi, Y. Akutsu. Colored Vertex Models, Colored IRF Models and Invariants of Trivalent Colored Graphs, J. Phys. Soc. Japan. 62 (1993) 19.

[36] J-H-H, Perk, C.L. Schultz, New families of commuting transfer matrices in $q$-state vertex models, Physics Letters A. 84 (1981) 407.

[37] H. Yamane, On Defining Relations of Affine Lie Superalgebras and Affine Quantized Universal Enveloping Superalgebras, Publ. RIMS. 35 (1999) 321.

[38] A. Hamel, R.C. King. Symplectic shifted tableaux and deformations of Weyl's denominator formula for $s p(2 n)$, J. Alg. Comb. 16 (2002) 269.

[39] A. Hamel, R.C. King. U-Turn Alternating Sign Matrices, Symplectic Shifted Tableaux and their Weighted Enumeration, J. Alg. Comb. 21 (2005) 395.

[40] S-Y. Zhao, Y-Z. Zhang. Supersymmetric vertex models with domain wall boundary conditions, J. Math. Phys. 48 (2007) 023504.

[41] A.D. Caradoc, O. Foda, M. Wheeler, M. Zuparic, On the trigonometric Felderhof model with domain wall boundary conditions, J. Stat. Mech. 2007 (2007) P03010.

[42] S-Y. Zhao, W-L.Yang, Y-Z. Zhang, Determinant representation of correlation functions for the $U_{q}(g l(1 \mid 1))$ free fermion model, J. Math. Phys. 47 (2006) 013302.

[43] D. Ivanov, Symplectic ice, in Multiple Dirichlet Series, L-Functions, and Automorphic Forms. D. Bump, S. Friedberg and D. Goldfield, eds., Progress in Mathematics. Birkhauser Boston, 300 (2012) 205-222.

[44] D. Ivanov, Part I, Symplectic ice, Part II, Global and local Kubota symbols. PhD. Thesis, Stanford University, USA (2010).

[45] S.J. Tabony, Deformations of characters, metaplectic Whittaker functions and the YangBaxter equation, PhD. Thesis, Massachusetts Institute of Technology, USA (2011).

[46] B. Brubaker, D. Bump, G. Chinta, P.E. Gunnells. Metaplectic functions and crystals of type B, in Multiple Dirichlet Series, L-Functions, and Automorphic Forms. D. Bump, S. Friedberg and D. Goldfield, eds., Progress in Mathematics. Birkhauser Boston, 300 (2012) 93-118.

[47] B. Brubaker, A. Schultz, The 6-vertex model and deformations of the Weyl character formula, J. Alg. Comb. 42 (2015) 917.

[48] B. Brubaker, V. Buciumas, D. Bump, A Yang-Baxter equation for metaplectic ice, Communications in Number Theory and Physics. 13 (2019) 101. 
[49] B. Brubaker, V. Buciumas, D. Bump, N. Gray, Duality for metaplectic ice, Appendix to [48.

[50] B. Brubaker, V. Buciumas, D. Bump, S. Friedberg, Hecke modules from metaplectic ice, Sel. Math. New Series 24 (2018) 2523.

[51] N. Gray, Metaplectic ice for Cartan type $C$, arXiv:1709.04971.

[52] B. Brubaker, V. Buciumas, D. Bump, H. Gustafsson, Vertex operators, solvable lattice models and metaplectic Whittaker functions, arXiv:1806.07776.

[53] K. Motegi, Dual wavefunction of the Felderhof model, Lett. Math. Phys. 107 (2017) 1235.

[54] K. Motegi, Izergin-Korepin Analysis on the Projected Wavefunctions of the Generalized Free-Fermion Model, Adv. Math. Phys. 2017 (2017) Article ID 7563781.

[55] K. Motegi, Elliptic supersymmetric integrable model and multivariable elliptic functions, Prog. Theor. Exp. Phys. 2017 (2017) 123A01.

[56] O. Foda, M. Wheeler, M. Zuparic, Two elliptic height models with factorized domain wall partition functions, J. Stat. Mech. 2008 (2008) P02001.

[57] M. Okado, Solvable Face Models Related to the Lie Superalgebra $s l(m \mid n)$, Lett. Math. Phys. 22 (1991) 39.

[58] T. Deguchi, A Fujii, IRF models associated with representations of the Lie superalgebras $g l(m \mid n)$ and $s l(m \mid n)$, Mod. Phys. Lett. A. 6 (1991) 3413.

[59] T. Deguchi, P. Martin, An algebraic approach to vertex models and transfer matrix spectra, Int. J. Mod. Phys. A. 7, no. suupp01a (1992) 165.

[60] D. Bump, S. Friedberg, J. Hoffstein, p-adic Whittaker functions on the metaplectic group, Duke Math. J. 63 (1991) 379.

[61] M. Wheeler, An Izergin-Korepin procedure for calculating scalar products in the sixvertex model, Nuclear Phys. B 852 (2011) 468.

[62] O. Tsuchiya, Determinant formula for the six-vertex model with reflecting end, J. Math. Phys. 39 (1998) 5946.

[63] G. Filali, N. Kitanine, The partition function of the trigonometric SOS model with a reflecting end, J. Stat. Mech. 2010 (2010) L06001.

[64] G. Filali, Elliptic dynamical reflection algebra and partition function of SOS model with reflecting end, J. Geom. Phys. 61 (2011) 1789.

[65] G.A.P. Ribeiro, V.E. Korepin, Thermodynamic limit of the six-vertex model with reflecting end, J. Phys. A: Math. and Theor. 48 (2015) 045205.

[66] N. Crampe, K. Mallick, E. Ragoucy, M. Vanicat. Inhomogeneous discrete-time exclusion processes, J. Phys. A: Math. and Theor. 48 (2015) 484002. 
[67] K. Motegi, Dual wavefunction of the symplectic ice, Rep. Math. Phys. 80 (2017) 414.

[68] K. Motegi, Izergin-Korepin analysis on the wavefunctions of the $U_{q}\left(s l_{2}\right)$ six-vertex model with reflecting end, to appear in Annales de l'Institut Henri Poincaré D.

[69] W-L. Yang, X. Chen, J. Feng, K. Hao, K-J. Shi, C-Y. Sun, Z-Y. Yang, Y-Z. Zhang, Domain wall partition function of the eight-vertex model with a non-diagonal reflecting end, Nuclear Phys. B 847 (2011) 367.

[70] W-L. Yang, X. Chen, J. Feng, K. Hao, K. Wu, Z-Y. Yang, Y-Z. Zhang, Scalar products of the open XYZ chain with non-diagonal boundary terms, Nuclear Phys. B 848 (2011) 523.

[71] W. Galleas, Multiple integral representation for the trigonometric SOS model with domain wall boundaries, Nuclear Phys. B 858 (2012) 117.

[72] W. Galleas, J. Lamers, Reflection algebra and functional equations, Nuclear Phys. B 886 (2014) 1003.

[73] J. Lamers, Integral formula for elliptic SOS models with domain walls and a reflecting end, Nuclear Phys. B 901 (2015) 556.

[74] S. Pakuliak, V. Rubtsov, A. Silantyev, The SOS model partition function and the elliptic weight functions, J. Phys. A: Math. and Theor. 41 (2008) 295204.

[75] H. Rosengren, An Izergin-Korepin-type identity for the 8VSOS model, with applications to alternating sign matrices, Adv. Appl. Math. 43 (2009) 137.

[76] W-L. Yang and Y-Z. Zhang, Partition function of the eight-vertex model with domain wall boundary condition, J. Math. Phys. 50 (2009) 083518.

[77] E. Sklyanin, Boundary conditions for integrable quantum systems, J. Phys. A: Math. Gen. 21 (1988) 2375.

[78] A. Morris, Spin representation of a direct sum and a direct product, J. London Math. Soc. 33 (1958) 326.

[79] R.C. King, Branching rules for classical Lie groups using tensor and spinor methods, J. Phys. A: Math. and Gen. 8 (1975) 429.

[80] M. Jimbo and T. Miwa, On a duality of branching rules for affine Lie algebras, Adv. Stud. in Pure Math. 6 (1985) 17.

[81] K. Hasegawa, Spin module versions of Weyl's reciprocity theorem for classical KacMoody Lie algebras - An application to branching rule duality, Publ. RIMS. 25 (1989) 741.

[82] I. Terada, A Robinson-Schensted-Type Correspondence for a Dual Pair on Spinors, J. Comb. Theory, Series A. 63 (1993) 90.

[83] D. Bump, A. Gamburd, On the Averages of Characteristic Polynomials From Classical Groups, Comm. Math. Phys. 265 (2006) 227. 
[84] A. Hamel and R.C. King, Bijective proof of a symplectic dual pair identity, SIAM Journal on Discrete Math. 25 (2011) 539. 